\title{
RELAXATION SCHEMES FOR THE MULTICOMPONENT EULER SYSTEM
}

\author{
StÉPHANe Dellacherie ${ }^{1}$
}

\begin{abstract}
We show that it is possible to construct a class of entropic schemes for the multicomponent Euler system describing a gas or fluid homogeneous mixture at thermodynamic equilibrium by applying a relaxation technique. A first order Chapman-Enskog expansion shows that the relaxed system formally converges when the relaxation frequencies go to the infinity toward a multicomponent Navier-Stokes system with the classical Fick and Newton laws, with a thermal diffusion which can be assimilated to a Soret effect in the case of a fluid mixture, and with also a pressure diffusion or a density diffusion respectively for a gas or fluid mixture. We also discuss on the link between the convexity of the entropies of each species and the existence of the Chapman-Enskog expansion.
\end{abstract}

Mathematics Subject Classification. 35Q30, 65M06, 76N10, 76T05, 80A15.

Received April 4, 2003.

\section{INTRODUCTION}

The aim of this article is to show that it is possible to construct a class of entropic schemes for gas and fluid mixtures flows described by the (monodimensionnal) multicomponent Euler system

$$
\left\{\begin{array}{l}
\forall k \in\{1, \ldots, \bar{k}-1\}: \partial_{t}\left(Y_{k} \rho\right)+\partial_{x}\left(Y_{k} \rho u\right)=0 \\
\partial_{t} \rho+\partial_{x}(\rho u)=0 \\
\partial_{t}(\rho u)+\partial_{x}\left(\rho u^{2}+P\right)=0 \\
\partial_{t}(\rho E)+\partial_{x}[(\rho E+P) u]=0 \\
E=\frac{1}{2} u^{2}+\varepsilon \\
\forall k: P_{k}=P_{k}\left(\tau_{k}, \varepsilon_{k}\right) \\
\sum_{k} Y_{k}=1
\end{array}\right.
$$

by using the ideas initially proposed by Coquel and Perthame in [7] (see also [1,17] and [10]). In this article, we use the following classical definitions: $\rho, P, u, \varepsilon$ and $E$ are the density, the pressure, the velocity, the internal and the total energies of the mixture (we also define the specific volume $\tau$ of the mixture with $\tau \equiv \rho^{-1}$ ); $Y_{k}$ is the mass fraction of the $k$ th species of the $\bar{k}$ species of the mixture; $P_{k}=P_{k}\left(\tau_{k}, \varepsilon_{k}\right)$ is the equation of state of the species $k$ where $P_{k}, \tau_{k}$ and $\varepsilon_{k}$ are the pressure, the specific volume and the internal energy of the species $k$ (we also define the density $\rho_{k}$ of the species $k$ with $\rho_{k} \equiv \tau_{k}^{-1}$ ). Let us remark that the system (1) implicitly

Keywords and phrases. Multicomponent Euler system, relaxation scheme, entropic scheme, Chapman-Enskog expansion.

1 Commissariat à l'Énergie Atomique, 91191 Gif-sur-Yvette, France. e-mail: stephane.dellacherie@cea.fr 
supposes that the isovelocity closure

$$
\forall\left(k, k^{\prime}\right): u_{k}=u_{k^{\prime}}
$$

is verified in the mixture. In the sequel, we will have to define other closure laws for the mixture pressure and for the mixture temperature to close the system (1). These supplementary closure laws will depend on the type of mixture which will be considered.

In this paper, we study two types of mixture that we name gas mixture and fluid mixture:

- a gas mixture is an intimate mixture where all the species occupy the entire volume for any infinitesimal volume;

- a fluid mixture is an immiscible mixture where all the species occupy different volumes from a mesoscopic point of view. Let us notice that this type of mixture could also be defined as a separated phase mixture.

The case of gas mixtures is classical in aerodynamics (see [20,21] and [9]) and in the field of the Boltzmann kinetic theory (see [4]). The case of (immiscible) fluid mixtures is also classical especially for the simulation of interfaces problems ( $c f$. $[2,3,16,18]$ and the references herein). Nevertheless, the present paper is not dedicated to simulations of interfaces problems where the fluid mixture is numerical and located to material interfaces, but to simulations of physical fluid mixtures at thermodynamic equilibrium (as in the case of gas mixtures) and at scales where it is impossible to define any material interface between each fluid. At last, let us remark that a similar approach of what is proposed in that paper is studied in [6] for the homentropic multicomponent Euler system.

The plan of this paper is the following: in Section 2, we define the closure laws to close the Euler system (1) for a gas mixture and a fluid mixture, we recall some important results on the hyperbolicity of (1) and we prove a Gibbs lemma for the mixture entropy; in Section 3, we define the relaxed multicomponent Euler system and the relaxation scheme, and we prove entropic results; in Section 4, we compute a Chapman-Enskog expansion to formally recover a classical multicomponent Navier-Stokes system from the relaxed system; at last, we conclude the paper.

\section{SOME PRELIMINARY RESULTS}

We define in this section the mixture laws for a gas mixture and for a fluid mixture, and we close the multicomponent Euler system by imposing closure laws on the pressure and on the temperature of the mixture. The closure laws are such that the mixture is always at thermodynamic equilibrium. After, we prove that the multicomponent Euler system (1) with the previous closure laws admits a convex entropy and is hyperbolic. At last, we prove a minimization principle - i.e. a Gibbs lemma - on the mixture entropy, result which will allow us to prove the entropic results for the numerical schemes in Section 3.

\subsection{Mixture and closure laws}

To close the system (1) constituted with $2 \bar{k}+4$ equations and with $4 \bar{k}+5$ unknowns, we need $2 \bar{k}+1$ closure laws. In the case of a gas mixture, we use the following $\bar{k}+2$ closure laws:

$$
\begin{cases}\varepsilon=\sum_{k} Y_{k} \varepsilon_{k}, & \text { (a) } \\ P=\sum_{k} P_{k}, & \text { (b) } \\ \forall k: Y_{k} \tau_{k}=\tau . & \text { (c) }\end{cases}
$$

Let us notice that $(3 \mathrm{~b})$ is the Dalton law which is a natural closure for a gas mixture.

For a fluid mixture, we use the following $\bar{k}+2$ closure laws:

$$
\left\{\begin{array}{l}
\varepsilon=\sum_{k} Y_{k} \varepsilon_{k}, \\
\forall\left(k, k^{\prime}\right): P_{k}=P_{k^{\prime}} \text { and } P=P_{k}, \quad \text { (b) } \\
\sum_{k} Y_{k} \tau_{k}=\tau .
\end{array}\right.
$$

The relation (4b) is the isobaric closure which is often used for fluid mixtures. 
The closure relations (3c) and (4c) are mixture laws which respectively impose that a gas mixture is an intimate mixture and that a fluid mixture is an immiscible mixture: in these two cases, we can define the notion of volumic fraction with

which imposes that

$$
\alpha_{k} \equiv \frac{Y_{k} \rho}{\rho_{k}}=\frac{Y_{k} \tau_{k}}{\tau}
$$

$$
\sum_{k} \alpha_{k} \rho_{k}=\rho
$$

since $\sum_{k} Y_{k}=1$. Moreover, the mixture law (3c) imposes that

$$
\forall k: \quad \alpha_{k}=1 \quad \text { for a gas mixture }
$$

which is coherent with the intimate character of the mixture; on the other hand, the mixture law (4c) is equivalent to

$$
\sum_{k} \alpha_{k}=1 \text { for a fluid mixture }
$$

and we have a priori $\alpha_{k} \neq \alpha_{k^{\prime}}$ which is a translation of the immiscible character of the mixture.

To obtain the last $\bar{k}-1$ closure laws, we use a thermodynamic hypothesis:

Hypothesis 2.1. Each equation of state $P_{k}\left(\tau_{k}, \varepsilon_{k}\right)$ admits a convex entropy $s_{k}\left(\tau_{k}, \varepsilon_{k}\right)$ and a thermodynamic temperature $T_{k}$ such that the second thermodynamic law

$$
-T_{k} \mathrm{~d} s_{k}=\mathrm{d} \varepsilon_{k}+P_{k} \mathrm{~d} \tau_{k}
$$

is verified.

Then, we are able to define the last $\bar{k}-1$ closure laws by imposing that

$$
\forall\left(k, k^{\prime}\right): T_{k}=T_{k^{\prime}}
$$

for gas and fluid mixtures. The closure law (10) corresponds to the isothermal closure. Let us notice that it is possible to define other closure laws as in $[12,16]$. Nevertheless, only the closure laws (3)-(10) and (4)-(10) will allow us to obtain entropic results with the relaxation scheme proposed in this paper.

Having defined the temperatures $T_{k}$ with (9), the closure relation (10) allows us to define the mixture temperature $T$ by taking $T \equiv T_{k}$ : we will see below that $T$ is also a thermodynamic temperature of the mixture. Moreover, we can now define the equations of state with $\tau_{k} \equiv \tau_{k}\left(T_{k}, P_{k}\right)$ and $\varepsilon_{k} \equiv \varepsilon_{k}\left(T_{k}, P_{k}\right)$. Let us remark that (1)-(3)-(10) and (1)-(4)-(10) are equivalent as soon as

$$
\forall k, \quad \exists \zeta_{k}\left(T_{k}\right) \quad \text { such that } \quad \tau_{k}\left(T_{k}, P_{k}\right)=\frac{\zeta_{k}\left(T_{k}\right)}{P_{k}} \quad \text { and } \quad \varepsilon_{k}\left(T_{k}, P_{k}\right)=\mathcal{E}_{k}\left(T_{k}\right)
$$

which is the case when all the species $k$ are ideal gases.

\subsection{Mixture entropy and hyperbolicity}

The multicomponent Euler system (1) being now closed, we are able to write the following result:

Theorem 2.1. Under the Hypothesis 2.1, the following properties are verified:

i) For any $\left\{Y_{k}\right\}_{k}, \tau$ and $\varepsilon$ in $\mathbb{R}^{+}$, the algebraic systems

$$
\left\{\begin{array}{l}
\forall k: Y_{k} \tau_{k}\left(T, P_{k}\right)=\tau, \\
\sum_{k} Y_{k} \varepsilon_{k}\left(T, P_{k}\right)=\varepsilon
\end{array} \quad\right. \text { (gas mixture) }
$$


and

$$
\left\{\begin{array}{l}
\sum_{k} Y_{k} \tau_{k}(T, P)=\tau, \\
\sum_{k} Y_{k} \varepsilon_{k}(T, P)=\varepsilon
\end{array} \quad\right. \text { (fluid mixture) }
$$

admit an unique solution respectively in $\left(\mathbb{R}^{+}\right)^{\bar{k}+1}$ and in $\left(\mathbb{R}^{+}\right)^{2}$.

ii) The multicomponent Euler system (1) is closed with the laws (3)-(10) for a gas mixture and with the laws (4)-(10) for a fluid mixture and is hyperbolic.

In the case of a fluid mixture, Theorem 2.1 was proven by Lagoutière in [16]; then, we have extended its proof to a case of a gas mixture in [12] by using similar techniques. More precisely, the proof of point $i i$ is based on an extension of Godunov-Mock theorem proven by Lagoutière in [16] which links the hyperbolicity of (1) to the existence of a mixture entropy $s\left(\left\{Y_{k}\right\}_{k}, \varepsilon, \tau\right)$ which is strictly convex with respect to $\varepsilon$ and $\tau$ for a fixed $\left\{Y_{k}\right\}_{k}$. This entropy is defined in the following proposition:

Proposition 2.1. Under the closure laws (3)-(10) or under the closure laws (4)-(10), the mixture entropy

$$
s \equiv \sum_{k} Y_{k} s_{k}
$$

verifies the following properties:

i) $s$ is a function of $\tau$ and $\varepsilon$ (and of course of $\left\{Y_{k}\right\}_{k}$ );

ii) $s\left(\left\{Y_{k}\right\}_{k}, \tau, \varepsilon\right)$ is strictly convex when $\left\{Y_{k}\right\}_{k}$ is fixed;

iii) when $d Y_{k}=0$, the mixture temperature $T$ verifies

$$
-T \mathrm{~d} s=\mathrm{d} \varepsilon+P \mathrm{~d} \tau .
$$

Thus, $T$ is the mixture thermodynamic temperature.

The proof is written in [12] for a gas mixture and in [16] for a fluid mixture.

\subsection{Gibbs lemma}

At last, we end this section with the important minimization principle which will allow us to prove in Section 3 the entropic properties of the relaxation scheme:

Proposition 2.2 (Gibbs Lemma). Let us suppose that $\left(\left\{Y_{k}\right\}, \tau, u, E\right)$ is known. Then, the mixture entropy $s\left(\left\{Y_{k}\right\}_{k}, \tau, \varepsilon\right)$ given by (14) verifies the minimization principle:

i) for a gas mixture described with the closure laws (3)-(10):

$$
s\left(\left\{Y_{k}\right\}_{k}, \tau, \varepsilon\right)=\min _{\left(\tau_{k}, u_{k}, \varepsilon_{k}\right)} \sum_{k} Y_{k} s_{k}\left(\tau_{k}, \varepsilon_{k}\right)
$$

under the constraints

$$
\left\{\begin{array}{l}
\forall k: Y_{k} \tau_{k}=\tau, \\
\sum_{k} Y_{k} u_{k}=u, \\
\sum_{k} Y_{k}\left(\varepsilon_{k}+\frac{1}{2} u_{k}^{2}\right)=E
\end{array}\right.
$$

and this minimum is reached at an unique point which is, as expected, the isothermal-isovelocity equilibrium deduced from system (12); 
ii) for a fluid mixture described with the closure laws (4)-(10):

$$
s\left(\left\{Y_{k}\right\}_{k}, \tau, \varepsilon\right)=\min _{\left(\tau_{k}, u_{k}, \varepsilon_{k}\right)} \sum_{k} Y_{k} s_{k}\left(\tau_{k}, \varepsilon_{k}\right)
$$

under the constraints

$$
\left\{\begin{array}{l}
\sum_{k} Y_{k} \tau_{k}=\tau \\
\sum_{k} Y_{k} u_{k}=u \\
\sum_{k} Y_{k}\left(\varepsilon_{k}+\frac{1}{2} u_{k}^{2}\right)=E
\end{array}\right.
$$

and this minimum is reached at an unique point which is, as expected, the isothermal-isobaric-isovelocity equilibrium deduced from system (13).

This proposition is similar to the Gibbs lemma which states - in its most classical form - that a Maxwellian distribution $\mathcal{M}(v)$ minimizes the quantity $\mathcal{H}(f) \equiv \int f(v) \log f(v) \mathrm{d} v$ under the constraint $\int f(v)\left(1, v, v^{2} / 2\right) \mathrm{d} v=$ Const., lemma which is very important in the kinetic theory (see [11] and the references herein for example): here, the Maxwellian distribution $\mathcal{M}(v)$ corresponds to the isothermal-isovelocity equilibrium for the gas mixture and to the isothermal-isobaric-isovelocity equilibrium for the fluid mixture, and the kinetic entropy $\mathcal{H}(f)$ corresponds to the mixture entropy $s \equiv \sum_{k} Y_{k} s_{k}$.

Proof of Proposition 2.2. Let us define $\sigma_{k}\left(\tau_{k}, u_{k}, E_{k}\right)=s_{k}\left(\tau_{k}, E_{k}-\frac{1}{2} u_{k}^{2}\right)$ and $\sigma\left(\left\{Y_{k}\right\}_{k}, \tau, u, E\right)=s\left(\left\{Y_{k}\right\}_{k}, \tau, E-\right.$ $\left.\frac{1}{2} u^{2}\right)$, and let us show that $\sigma\left(\left\{Y_{k}\right\}_{k}, \tau, u, E\right)=\min _{\left(\tau_{k}, u_{k}, E_{k}\right)} \sum_{k} Y_{k} \sigma_{k}\left(\tau_{k}, u_{k}, E_{k}\right)$. Since for all $k, \sigma_{k}\left(\tau_{k}, u_{k}, E_{k}\right)$ is strictly convex because of the convexity of $s_{k}\left(\tau_{k}, \varepsilon_{k}\right)\left(c f\right.$. Lem. 1.2, p. 101 in [14]), $\sum_{k} Y_{k} \sigma_{k}$ is also strictly convex (we recall that $\left\{Y_{k}\right\}_{k}$ is fixed). Then, we minimize a strictly convex function under linear constraints which induces that we just have to prove the existence of a local minimum to obtain the existence of an unique global minimum.

So, let us verify that the isothermal-isovelocity equilibrium - solution of (12) - is a local minimum for a gas mixture and that the isothermal-isobaric-isovelocity equilibrium - solution of (13) - is a local minimum for a fluid mixture. We define this equilibrium with $\mathcal{U}^{0}=\left\{\left(\tau_{k}^{0}, u_{k}^{0}, E_{k}^{0}\right)\right\}_{k}$ and we recall that it is unique (cf. the point $i$ of Th. 2.1). The Taylor expansion of $\sum_{k} Y_{k} \sigma_{k}\left(\tau_{k}, u_{k}, E_{k}\right)$ around $\mathcal{U}^{0}$ gives

$$
\sum_{k} Y_{k} \sigma_{k}\left(\tau_{k}^{0}+\delta \tau_{k}, u_{k}^{0}+\delta u_{k}, E_{k}^{0}+\delta E_{k}\right)=\sum_{k} Y_{k} \sigma_{k}\left(\tau_{k}^{0}, u_{k}^{0}, E_{k}^{0}\right)+\frac{\mathrm{d} \sigma}{\mathrm{d} \mathcal{U}^{0}} \delta \mathcal{U}+\text { second order term }
$$

where

$$
\frac{\mathrm{d} \sigma}{\mathrm{d} \mathcal{U}^{0}} \delta \mathcal{U}=\sum_{k} Y_{k}\left[\frac{\partial \sigma_{k}}{\partial \tau_{k}}\left(\tau_{k}^{0}, u_{k}^{0}, E_{k}^{0}\right) \delta \tau_{k}+\frac{\partial \sigma_{k}}{\partial u_{k}}\left(\tau_{k}^{0}, u_{k}^{0}, E_{k}^{0}\right) \delta u_{k}+\frac{\partial \sigma_{k}}{\partial E_{k}}\left(\tau_{k}^{0}, u_{k}^{0}, E_{k}^{0}\right) \delta E_{k}\right] .
$$

We know that the second order term is strictly positive because of the positivity of the Hessian matrix of each $\sigma_{k}$. Thus, we just have to show that the first order term $\frac{\mathrm{d} \sigma}{\mathrm{d} \mathcal{U}^{0}} \delta \mathcal{U}$ is non negative to obtain the final result. First of all, let us notice that

$$
\frac{\mathrm{d} \sigma}{\mathrm{d} \mathcal{U}^{0}} \delta \mathcal{U}=\sum_{k}\left(-\frac{P_{k}^{0}}{T_{k}^{0}} Y_{k} \delta \tau_{k}\right)+\sum_{k}\left(\frac{u_{k}^{0}}{T_{k}^{0}} Y_{k} \delta u_{k}\right)+\sum_{k}\left(-\frac{1}{T_{k}^{0}} Y_{k} \delta E_{k}\right)
$$

since

$$
\left\{\begin{array}{l}
\frac{\partial \sigma_{k}}{\partial \tau_{k}}\left(\tau_{k}^{0}, u_{k}^{0}, E_{k}^{0}\right)=\frac{\partial s_{k}}{\partial \tau_{k}}\left(\tau_{k}^{0}, \varepsilon_{k}^{0}\right)=-\frac{P_{k}^{0}}{T_{k}^{0}} \\
\frac{\partial \sigma_{k}}{\partial u_{k}}\left(\tau_{k}^{0}, u_{k}^{0}, E_{k}^{0}\right)=-u_{k}^{0} \frac{\partial s_{k}}{\partial \varepsilon_{k}}\left(\tau_{k}^{0}, \varepsilon_{k}^{0}\right)=\frac{u_{k}^{0}}{T_{k}^{0}} \\
\frac{\partial \sigma_{k}}{\partial E_{k}}\left(\tau_{k}^{0}, u_{k}^{0}, E_{k}^{0}\right)=\frac{\partial s_{k}}{\partial \varepsilon_{k}}\left(\tau_{k}^{0}, \varepsilon_{k}^{0}\right)=-\frac{1}{T_{k}^{0}}
\end{array}\right.
$$


Secondly, since the equilibrium $\mathcal{U}^{0}$ is an isothermal-isovelocity equilibrium, we have $T_{k}^{0} \equiv T^{0}$ and $u_{k}^{0} \equiv u^{0}$; moreover, the constraints $(16 \mathrm{~b}, \mathrm{c})$ or $(18 \mathrm{~b}, \mathrm{c})$ induce that $\sum_{k} Y_{k} \delta u_{k}=0$ and $\sum_{k} Y_{k} \delta E_{k}=0$. So, we obtain that

$$
\frac{\mathrm{d} \sigma}{\mathrm{d} \mathcal{U}^{0}} \delta \mathcal{U}=-\frac{1}{T^{0}} \sum_{k} P_{k}^{0} Y_{k} \delta \tau_{k} .
$$

In the case of a gas mixture, the constraint (16a) induces that for all $k, Y_{k} \delta \tau_{k}=0$; in the case of a fluid mixture, the isobaric closure law (4b) and the constraint (18a) respectively induces that $P_{k}^{0} \equiv P^{0}$ and $\sum_{k} Y_{k} \delta \tau_{k}=0$. Then, in each case, we find that $\frac{\mathrm{d} \sigma}{\mathrm{d} \mathcal{U}^{0}} \delta \mathcal{U}=0$ which concludes the proof.

\section{Relaxation SCHEME AND ENTROpiC RESUlts}

The aim of this section is to propose a class of entropic schemes solving the multicomponent Euler system (1) closed with (3)-(10) for a gas mixture and closed with (4)-(10) for a fluid mixture. The key point of the construction of these schemes is to "artificially separate" the two species by introducing a relaxed multicomponent Euler system and, then, by solving the mono-species Euler system for each species before relaxing the mixture to a thermodynamic equilibrium mixture i.e. such that the closure relations (2)-(10) and the closure relations (3) or $(4)$ are verified. We will see that by using the minimization principles $(15,16)$ and $(17,18)$, this procedure will allow us to construct a class of entropic schemes under a CFL criterion and to recover some entropic results on existing numerical schemes.

Let us notice that the relaxed system studied in that paper is similar but not exactly equal to the one proposed in [2].

To simplify the notations, we write the results for a mixture of two species (i.e. for a binary mixture) and the discretization of the equations is written for a Cartesian mono-dimensional geometry.

\subsection{The relaxed system}

Let us write the relaxed system

$$
\left\{\begin{aligned}
& \partial_{t}\left(\alpha_{1} \rho_{1}\right)+\partial_{x}\left(\alpha_{1} \rho_{1} u_{1}\right)=0 \\
& \partial_{t}\left(\alpha_{1} \rho_{1} u_{1}\right)+\partial_{x}\left(\alpha_{1} \rho_{1} u_{1}^{2}+\alpha_{1} P_{1}\right)=\frac{1}{\lambda_{u}}\left(u_{2}-u_{1}\right)+\kappa P_{\text {int }} \partial_{x} \alpha_{1} \\
& \partial_{t}\left(\alpha_{1} \rho_{1} E_{1}\right)+\partial_{x}\left[\left(\alpha_{1} \rho_{1} E_{1}+\alpha_{1} P_{1}\right) u_{1}\right]= \frac{1}{\lambda_{T}}\left(T_{2}-T_{1}\right)+\frac{U_{\text {inter }}}{\lambda_{u}}\left(u_{2}-u_{1}\right) \\
&+\kappa\left[P_{\text {int }} U_{\text {int }} \partial_{x} \alpha_{1}+\frac{P_{\text {inter }}}{\lambda_{P}}\left(P_{2}-P_{1}\right)\right] \\
& \partial_{t}\left(\alpha_{2} \rho_{2}\right)+\partial_{x}\left(\alpha_{2} \rho_{2} u_{2}\right)=0, \frac{1}{\lambda_{u}}\left(u_{1}-u_{2}\right)+\kappa P_{\text {int }} \partial_{x} \alpha_{2}, \\
& \partial_{t}\left(\alpha_{2} \rho_{2} u_{2}\right)+\partial_{x}\left(\alpha_{2} \rho_{2} u_{2}^{2}+\alpha_{2} P_{2}\right)= \\
& \partial_{t}\left(\alpha_{2} \rho_{2} E_{2}\right)+\partial_{x}\left[\left(\alpha_{2} \rho_{2} E_{2}+\alpha_{2} P_{2}\right) u_{2}\right]= \frac{1}{\lambda_{T}}\left(T_{1}-T_{2}\right)+\frac{U_{\text {inter }}}{\lambda_{u}}\left(u_{1}-u_{2}\right) \\
&+\kappa\left[P_{\text {int }} U_{\text {int }} \partial_{x} \alpha_{2}+\frac{P_{\text {inter }}}{\lambda_{P}}\left(P_{1}-P_{2}\right)\right]
\end{aligned}\right.
$$

where the temperature $T_{k}$ is the thermodynamic temperature associated to the equation of state $P_{k}\left(\tau_{k}, \varepsilon_{k}\right)$ of the $k$ th species through the Hypothesis 2.1. We recall that $\alpha_{k}$ is the volumic fraction of the species $k$ and verifies (7) and (8) respectively for a gas and fluid mixture. The constant $\kappa$ is such that $\kappa=0$ for a gas mixture and $\kappa=1$ for a fluid mixture: see below. 
This system models a gas or fluid mixture which is not at thermodynamic equilibrium which is the case when $P_{1} \neq P_{2}$ for a fluid mixture and when $u_{1} \neq u_{2}, T_{1} \neq T_{2}$ for a gas or fluid mixture. To force the mixture to go to the thermodynamic equilibrium, we introduce in the system (20) relaxation terms which are functions of $u_{2}-u_{1}, T_{2}-T_{1}$ and, in the case of a fluid mixture, are functions of $P_{2}-P_{1}$. The coefficients $\lambda_{u}, \lambda_{T}$ and $\lambda_{P}$ are strictly positive modelling parameters.

We call the quantities $U_{\text {inter }}$ and $P_{\text {inter }}$ respectively interfacial velocity and interfacial pressure in the case of a fluid mixture - as in [2] - and, in that paper, also for a gas mixture although the notion of interface between species disappears in a gas mixture: these quantities will be defined below ( $f$. (27) and (28)).

The parameter $\kappa$ is a constant belonging to $\{0,1\}$ which turns off or turns on the pressure relaxation according to the kind of mixture. Indeed, to take into account the Dalton law (3b) for a gas mixture and the isobaric closure law (4b) for a fluid mixture, we close the system (20) with:

- For a gas mixture: in that case, the thermodynamic equilibrium does not impose that $P_{1}$ is equal to $P_{2}$. Thus, we turn off the pressure relaxation by taking

$$
\kappa=0 .
$$

We easily verify that the system (20) is closed when each $\alpha_{k}$ is given by

$$
\left\{\begin{array}{l}
\alpha_{1}=1, \\
\alpha_{2}=1
\end{array}\right.
$$

which is the case for a gas mixture $(c f .(7))$.

- For a fluid mixture: the closure relation (4b) means that the system (20) has to include pressure relaxation phenomena to obtain a good thermodynamic equilibrium. Thus, we impose that

$$
\kappa=1
$$

and we close the system (20) with

$$
\left\{\begin{array}{l}
\alpha_{1}+\alpha_{2}=1 \quad(c f .(8)) \\
\partial_{t} \alpha_{1}+U_{\text {inter }} \partial_{x} \alpha_{1}=\frac{1}{\lambda_{P}}\left(P_{1}-P_{2}\right)
\end{array}\right.
$$

The equation (24b) will allow to obtain an H-theorem ( $c f$. Lem. 3.1) and will allow to perform a Chapman-Enskog expansion in Section 4 (see also Sect. A.3).

It is important to underline that, for a fluid mixture, the non conservative terms $P_{\text {int }} \partial_{x} \alpha_{k}$ and $P_{\text {int }} U_{\text {int }} \partial_{x} \alpha_{k}$ in (20) are not necessary to obtain a system which conserves the momentum and the total energy of the fluid mixture. From a physical point of view, they are supposed to model at a macroscopic level the mesoscopic phenomena at the physical interface between the immiscible fluids.

What we can precisely say in this paper is that they are necessary to obtain a Navier-Stokes system with a Chapman-Enskog expansion applied to the relaxed Euler system (20): see Section 4 and Section A.3 in Appendix A.

Let us notice that the system (20) is very similar to the one proposed by Abgrall and Saurel in [2] for a fluid mixture. The difference is that we take into account temperature relaxation phenomena contrary to what is done in [2]: this will allow us to do a Chapman-Enskog expansion in Section 4 which would be certainly more complicated without any temperature relaxation phenomena. 
Let us remark that the system $(20)$ closed with $(21,22)$ or with $(23,24)$ can be rewritten with

$$
\begin{aligned}
& \left\{\begin{aligned}
& \partial_{t} \rho_{1}+\partial_{x}\left(\rho_{1} u_{1}\right)=\kappa\left[\frac{\rho_{1}}{\alpha_{1} \lambda_{P}}\left(P_{2}-P_{1}\right)+\rho_{1}\left(U_{\text {inter }}-u_{1}\right) \frac{\partial_{x} \alpha_{1}}{\alpha_{1}}\right], \\
& \partial_{t}\left(\rho_{1} u_{1}\right)+\partial_{x}\left(\rho_{1} u_{1}^{2}+P_{1}\right)= \frac{1}{\alpha_{1} \lambda_{u}}\left(u_{2}-u_{1}\right) \\
&+\kappa\left\{\frac{\rho_{1}}{\alpha_{1} \lambda_{P}} u_{1}\left(P_{2}-P_{1}\right)+\left[\rho_{1} u_{1}\left(U_{\text {inter }}-u_{1}\right)+\left(P_{\text {int }}-P_{1}\right)\right] \frac{\partial_{x} \alpha_{1}}{\alpha_{1}}\right\},
\end{aligned}\right. \\
& \partial_{t}\left(\rho_{1} E_{1}\right)+\partial_{x}\left[\left(\rho_{1} E_{1}+P_{1}\right) u_{1}\right]=\frac{1}{\alpha_{1} \lambda_{T}}\left(T_{2}-T_{1}\right)+\frac{1}{\alpha_{1} \lambda_{u}} U_{\text {inter }}\left(u_{2}-u_{1}\right) \\
& +\kappa\left\{\frac{1}{\alpha_{1} \lambda_{P}}\left(\rho_{1} E_{1}+P_{\text {inter }}\right)\left(P_{2}-P_{1}\right)\right. \\
& \left.+\left[\left(\rho_{1} E_{1}+P_{1}\right)\left(U_{\mathrm{int}}-u_{1}\right)+U_{\mathrm{int}}\left(P_{\mathrm{int}}-P_{1}\right)\right] \frac{\partial_{x} \alpha_{1}}{\alpha_{1}}\right\}, \\
& \partial_{t} \rho_{2}+\partial_{x}\left(\rho_{2} u_{2}\right)=\kappa\left[\frac{\rho_{2}}{\alpha_{2} \lambda_{P}}\left(P_{1}-P_{2}\right)+\rho_{2}\left(U_{\text {inter }}-u_{2}\right) \frac{\partial_{x} \alpha_{2}}{\alpha_{2}}\right] \\
& \partial_{t}\left(\rho_{2} u_{2}\right)+\partial_{x}\left(\rho_{2} u_{2}^{2}+P_{2}\right)=\frac{1}{\alpha_{2} \lambda_{u}}\left(u_{1}-u_{2}\right) \\
& +\kappa\left\{\frac{\rho_{2}}{\alpha_{2} \lambda_{P}} u_{2}\left(P_{1}-P_{2}\right)+\left[\rho_{2} u_{2}\left(U_{\text {inter }}-u_{2}\right)+\left(P_{\text {int }}-P_{2}\right)\right] \frac{\partial_{x} \alpha_{2}}{\alpha_{2}}\right\}, \\
& \partial_{t}\left(\rho_{2} E_{2}\right)+\partial_{x}\left[\left(\rho_{2} E_{2}+P_{2}\right) u_{2}\right]=\frac{1}{\alpha_{2} \lambda_{T}}\left(T_{1}-T_{2}\right)+\frac{1}{\alpha_{2} \lambda_{u}} U_{\text {inter }}\left(u_{1}-u_{2}\right) \\
& +\kappa\left\{\frac{1}{\alpha_{2} \lambda_{P}}\left(\rho_{2} E_{2}+P_{\text {inter }}\right)\left(P_{1}-P_{2}\right)\right. \\
& \left.+\left[\left(\rho_{2} E_{2}+P_{2}\right)\left(U_{\mathrm{int}}-u_{2}\right)+U_{\mathrm{int}}\left(P_{\mathrm{int}}-P_{2}\right)\right] \frac{\partial_{x} \alpha_{2}}{\alpha_{2}}\right\}
\end{aligned}
$$

which is more complicated when $\kappa=1$ but does not rise $\alpha_{k}$ in the left hand sides of system. Of course, for a gas mixture (i.e. when $\alpha_{k}=1$ and $\kappa=0$ ), the system (25) is exactly the same than the system (20). The system (25) is important because this will be under that form that we will deduce the relaxation scheme for a fluid mixture.

We can notice that the non conservative complicated terms proportional to $\partial_{x} \alpha_{k} / \alpha_{k}$ in the right hand sides of (25), firstly, are due to the non conservative terms $P_{\text {int }} \partial_{x} \alpha_{k}$ and $P_{\text {int }} U_{\text {int }} \partial_{x} \alpha_{k}$ in (20) and, secondly, allow to conserve the mass of each fluid and the momentum and total energy of the fluid mixture.

The following result shows that the relaxed system (20) is formally coherent with the multicomponent Euler system (1) under conditions on $U_{\text {inter }}$ and $P_{\text {inter }}$ :

Property 3.1. Let us define

$$
\left\{\begin{array}{l}
\rho \equiv \alpha_{1} \rho_{1}+\alpha_{2} \rho_{2} \\
Y_{1} \equiv \alpha_{1} \rho_{1} / \rho \\
Y_{2} \equiv 1-Y_{1} \\
u \equiv Y_{1} u_{1}+Y_{2} u_{2} \\
E \equiv Y_{1} E_{1}+Y_{2} E_{2}
\end{array}\right.
$$


Then, under the Hypothesis 2.1, we have:

i) For a gas mixture: when $\lambda_{u}$ and $\lambda_{T}$ go to zero, the relaxed system (20) closed with (21, 22) is equivalent to the multicomponent Euler system (1) closed with (3)-(10) as soon as

$$
U_{\text {inter }} \in\left[\min \left(u_{1}, u_{2}\right), \max \left(u_{1}, u_{2}\right)\right]
$$

ii) For a fluid mixture: when $\lambda_{u}, \lambda_{T}$ and $\lambda_{P}$ go to zero, the relaxed system (20) closed with (23, 24) is equivalent to the multicomponent Euler system (1) closed with (4)-(10) as soon as

$$
\left\{\begin{array}{l}
U_{\text {inter }} \in\left[\min \left(u_{1}, u_{2}\right), \max \left(u_{1}, u_{2}\right)\right] \\
P_{\text {inter }} \in\left[\min \left(P_{1}, P_{2}\right), \max \left(P_{1}, P_{2}\right)\right]
\end{array}\right.
$$

In Section 4, we will precise this property by recovering a Navier-Stokes system with a Chapman-Enskog expansion applied to relaxed system (20).

To prove this formal property, we firstly prove that the equilibrium solution of spatially homogeneous relaxed system

$$
\left\{\begin{aligned}
& \partial_{t} \alpha_{1}=\frac{\kappa}{\lambda_{P}}\left(P_{1}-P_{2}\right), \\
& \partial_{t}\left(\alpha_{1} \rho_{1}\right)=0, \\
& \partial_{t}\left(\alpha_{1} \rho_{1} u_{1}\right)=\frac{1}{\lambda_{u}}\left(u_{2}-u_{1}\right), \\
& \partial_{t}\left(\alpha_{1} \rho_{1} E_{1}\right)=\frac{1}{\lambda_{T}}\left(T_{2}-T_{1}\right)+\frac{U_{\text {inter }}}{\lambda_{u}}\left(u_{2}-u_{1}\right) \\
& \quad+\kappa \frac{P_{\text {inter }}}{\lambda_{P}}\left(P_{2}-P_{1}\right), \\
& \partial_{t}\left(\alpha_{2} \rho_{2}\right)=0, \\
& \partial_{t}\left(\alpha_{2} \rho_{2} u_{2}\right)= \frac{1}{\lambda_{u}}\left(u_{1}-u_{2}\right), \\
& \partial_{t}\left(\alpha_{2} \rho_{2} E_{2}\right)= \frac{1}{\lambda_{T}}\left(T_{1}-T_{2}\right)+\frac{U_{\text {inter }}}{\lambda_{u}}\left(u_{1}-u_{2}\right) \\
&+\kappa \frac{P_{\text {inter }}}{\lambda_{P}}\left(P_{1}-P_{2}\right)
\end{aligned}\right.
$$

closed with $(21,22)$ or with $(23,24$ a) when $t$ goes to the infinity is respectively an isothermal-isovelocity equilibrium for a gas mixture or an isothermal-isobaric-isovelocity equilibrium for a fluid mixture:

Lemma 3.1. (H-theorem) The quantities $Y_{k}, \rho, u$ and $E$ defined by (26) and $\varepsilon \equiv E-u^{2} / 2$ are constants of dynamical system (29). Moreover, under the Hypothesis 2.1, the mixture entropy $s \equiv \sum_{k} Y_{k} s_{k}$ decreases and we have:

i) For a gas mixture: when $t$ goes to the infinity, the solution of (29) converges to the isothermal

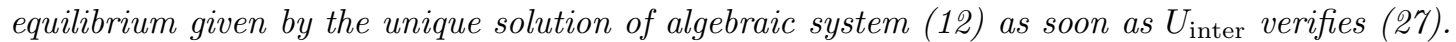

ii) For a fluid mixture: when $t$ goes to the infinity, the solution of (29) converges to the isothermalisobaric equilibrium given by the unique solution of algebraic system (13) as soon as $U_{\text {inter }}$ and $P_{\text {inter }}$ verify (28).

And, in these two cases, the velocities $u_{k}$ converge to the velocity $u$. Moreover, the final equilibrium minimizes the mixture entropy.

Let us notice that this lemma is the translation for the relaxed multicomponent Euler system (20) of the well known H-theorem of Boltzmann (1872) traditionally applied to kinetic equations (see [11] and the references herein for example). 
Proof of Lemma 3.1. Simple calculus show that $Y_{k}, \rho, u$ and $E$ are constants of (29). We show now that $s=\sum_{k} Y_{k} s_{k}$ verifies $\partial_{t} s \leq 0$ and that $\partial_{t} s=0$ if and only if the system has reached an unique equilibrium which minimizes $s$. Then, we will be able to conclude by using the Lyapounov theorem.

Since, by using (9), we have $-T_{1} \partial_{t} s_{1}=P_{1} \partial_{t} \tau_{1}+\partial_{t} E_{1}-u_{1} \partial_{t} u_{1}$, we easily find that

$$
-T_{1} \partial_{t} s_{1}=\frac{1}{Y_{1} \rho \lambda_{T}}\left(T_{2}-T_{1}\right)+\kappa \frac{\left(P_{\text {inter }}-P_{1}\right)}{Y_{1} \rho \lambda_{P}}\left(P_{2}-P_{1}\right)+\frac{U_{\text {inter }}-u_{1}}{Y_{1} \rho \lambda_{u}}\left(u_{2}-u_{1}\right)
$$

by using (29). In the same way, we obtain

$$
-T_{2} \partial_{t} s_{2}=\frac{1}{Y_{2} \rho \lambda_{T}}\left(T_{1}-T_{2}\right)+\kappa \frac{\left(P_{\text {inter }}-P_{2}\right)}{Y_{2} \rho \lambda_{P}}\left(P_{1}-P_{2}\right)+\frac{U_{\text {inter }}-u_{2}}{Y_{2} \rho \lambda_{u}}\left(u_{1}-u_{2}\right) .
$$

Since $\partial_{t} Y_{k}=0$, we have

$$
\partial_{t} s=\partial_{t}\left(Y_{1} s_{1}+Y_{2} s_{2}\right)=Y_{1} \partial_{t} s_{1}+Y_{2} \partial_{t} s_{2}
$$

and we obtain

$$
\begin{aligned}
\partial_{t} s= & \frac{\tau}{\lambda_{T}}\left(T_{2}-T_{1}\right)\left(\frac{1}{T_{2}}-\frac{1}{T_{1}}\right)+\frac{\tau}{\lambda_{u}}\left(u_{2}-u_{1}\right)\left[\frac{\left(U_{\text {inter }}-u_{2}\right)}{T_{2}}-\frac{\left(U_{\text {inter }}-u_{1}\right)}{T_{1}}\right] \\
& +\kappa \frac{\tau}{\lambda_{P}}\left(P_{2}-P_{1}\right)\left[\frac{\left(P_{\text {inter }}-P_{2}\right)}{T_{2}}-\frac{\left(P_{\text {inter }}-P_{1}\right)}{T_{1}}\right]
\end{aligned}
$$

Thus, when $U_{\text {inter }}$ and $P_{\text {inter }}$ verify (27) when $\kappa=0$ and (28) when $\kappa=1$, we find that $\partial_{t} s \leq 0$ and that $\partial_{t} s=0$ if and only if $u_{1}=u_{2}, T_{1}=T_{2}$ and (when $\left.\kappa=1\right) P_{1}=P_{2}$, equilibrium which is unique (cf. point $i$ of Th. 2.1) and which is the minimum of $s$ (cf. Prop. 2.2).

Formal proof of property 3.1. By using the Lemma 3.1, it is legitimate to state that when $\lambda_{u}, \lambda_{T}$ and $\lambda_{P}$ go to zero, the velocities, the temperatures and the pressures are given by the equilibrium of spatially homogeneous relaxed system (29). Then, by using the definition (26), we easily obtain the result by summing (20a,b,c) respectively with $\left(20 \mathrm{a}^{\prime}, \mathrm{b}^{\prime}, \mathrm{c}^{\prime}\right)$.

\subsection{The numerical scheme and the entropy properties}

The subscripts $n$ and $i$ are respectively the time and space subscripts and we recall that $k$ is the species subscript. The time and the mesh points are respectively defined by $t^{n}$ and $\left\{x_{i}\right\}_{i}$.

Definition of the relaxation scheme: Using the Property 3.1, it is legitimate to solve (1) by doing a splitting between the hydrodynamic part of (20) or of (25) and the spatially homogeneous relaxation part (29) of (20) solved by making $\lambda_{u}, \lambda_{T}$ and $\lambda_{P}$ converge to zero. Then, the construction of the relaxation scheme is the following:

\section{Hydrodynamic stage:}

Let us suppose that we know $\left(Y_{1}^{n}, \rho^{n}, u^{n}, E^{n}\right)$ at the time $t^{n}$ in each mesh point $x_{i}$. Of course, we know $\left(T^{n}, P_{1}^{n}, P_{2}^{n}\right)$ - and thus $\left(\alpha_{k}^{n}, \rho_{k}^{n}, u_{k}^{n}=u, E_{k}^{n}\right)$ - since the mixture is supposed to be at thermodynamic equilibrium at the time $t^{n}$.

The hydrodynamic stage consists in "artificially separating" each species $k$ of the mixture by solving with an explicit finite volume scheme coupled to the initial condition $\left(\rho_{k}^{n}, u_{k}^{n}=u^{n}, E_{k}^{n}\right)$ the hydrodynamic part of (20).

Since the mixture is at thermodynamic equilibrium at the time $t^{n}$ and since the scheme is explicit, we have under the conditions (27) or (28) that $u_{k}^{n}=U_{\text {int }}^{n}$ and, for a fluid mixture, $P_{k}^{n}=P_{\text {int }}^{n}$. As a consequence, the non conservative terms in the right hand side of (25) disappear for a fluid mixture (we recall that these terms do 
not exist for a gas mixture) which would not be the case by discretizing the hydrodynamic part of (20): thus, to define the hydrodynamic stage, we use the system (25) instead of a priori more simple system (20).

Thus, for a gas or fluid mixture, we solve in a time step $\Delta t$

$$
\left\{\begin{array}{l}
\partial_{t} \rho_{k}+\partial_{x}\left(\rho_{k} u_{k}\right)=0 \\
\partial_{t}\left(\rho_{k} u_{k}\right)+\partial_{x}\left(\rho_{k} u_{k}^{2}+P_{k}\right)=0, \\
\partial_{t}\left(\rho_{k} E_{k}\right)+\partial_{x}\left[\left(\rho_{k} E_{k}+P_{k}\right) u_{k}\right]=0
\end{array}\right.
$$

with an Eulerian explicit finite volume scheme or we solve

$$
\left\{\begin{array}{l}
D_{t}^{k} \tau_{k}=\partial_{m_{k}} u_{k}, \\
D_{t}^{k} u_{k}=-\partial_{m_{k}} P_{k}, \\
D_{t}^{k} E_{k}=-\partial_{m_{k}}\left(P_{k} u_{k}\right)
\end{array}\right.
$$

with a Lagrangian explicit finite volume scheme (knowing that $D_{t}^{k}=\partial_{t}+u_{k} \partial_{x}$ and $\partial_{m_{k}}=\tau_{k} \partial_{x}$ ) which gives $\left(\rho_{k}^{*}, u_{k}^{*}, E_{k}^{*}\right)$ at the intermediate time $t^{*}$. Let us notice that we have $u_{1}^{*} \neq u_{2}^{*}, T_{1}^{*} \neq T_{2}^{*}$ and, for a fluid mixture, $P_{1}^{*} \neq P_{2}^{*}$ since, at the time $t^{*}$, the mixture is not at thermodynamic equilibrium.

What is important to remark here is that we have "forgotten" the type of mixture at the time $t^{*}$ by solving (33) or (34) for each species $k$ : the aim of the following relaxation stage will be to "mix" the species together by taking into account the mixture laws (3c) or (4c).

\section{Relaxation stage:}

We "mix" the species together to obtain a thermodynamic equilibrium mixture by solving (29) in the same time step $\Delta t$ with the initial condition given by $\left(\rho_{k}^{*}, u_{k}^{*}, E_{k}^{*}\right)$ by making $\lambda_{u}, \lambda_{T}$ and $\lambda_{P}$ converge to zero. Then, because of the Lemma 3.1 , we obtain $\left(Y_{k}^{n+1}, \rho^{n+1}, u^{n+1}, E^{n+1}\right)$ in each mesh point at the time $t^{n+1}$ with

$$
\left\{\begin{array}{l}
Y_{k}^{n+1}=Y_{k}^{*}, \\
\rho^{n+1}=\rho^{*}, \\
u^{n+1}=Y_{1}^{*} u_{1}^{*}+Y_{2}^{*} u_{2}^{*}, \\
E^{n+1}=Y_{1}^{*} E_{1}^{*}+Y_{2}^{*} E_{2}^{*}
\end{array}\right.
$$

and we obtain $\left(\rho_{1}^{n+1}, \rho_{2}^{n+1}, T^{n+1}, P_{1}^{n+1}, P_{2}^{n+1}\right)$ by solving the system (12) for a gas mixture or the system (13) for a fluid mixture. Let us remark that a simple calculus shows that $\varepsilon^{n+1} \equiv E^{n+1}-\frac{1}{2}\left(u^{n+1}\right)^{2}$ is strictly positive as soon as $Y_{k}^{*}>0$ and $\varepsilon_{k}^{*}>0$.

Condition to obtain an entropic relaxation scheme: We will see in Section 3.2.1 and in Section 3.2.2 that a condition to obtain entropic results is that the previous relaxation numerical scheme verifies the following hypothesis:

Hypothesis 3.1. The explicit finite volume schemes used to solve (33) or (34) defined with the numerical flux $f_{k}=\left(f_{k, 1}, f_{k, 2}, f_{k, 3}\right)$ for each species $k$ in Eulerian or Lagrangian variables are entropic under a CFL criterion.

\subsubsection{For a gas mixture in Eulerian variables}

Let us now define the Eulerian finite volume scheme

$$
U_{i}^{n+1}=U_{i}^{n}-\frac{\Delta t}{\Delta x_{i}}\left[f(U)_{i+\frac{1}{2}}^{n}-f(U)_{i-\frac{1}{2}}^{n}\right]
$$


for the multicomponent Euler system (1) closed by (3)-(10) with $U=\left(Y_{1} \rho, \rho, \rho u, \rho E\right), f(U)_{i+\frac{1}{2}}^{n}$ being an $a d$ hoc numerical hydrodynamic flux defined in the following theorem. The subscript of the interface between the mesh point $x_{i}$ and $x_{i+1}$ is noted $i+1 / 2$ and the time and space steps are respectively noted $\Delta t$ and $\Delta x_{i}$.

We have the following result:

Theorem 3.1. Let us suppose that the numerical hydrodynamic flux $f(U)_{i+\frac{1}{2}}^{n}$ in (36) is simply defined by

$$
f(U)_{i+\frac{1}{2}}^{n}=\left(\begin{array}{c}
f_{1,1}\left(U_{1}\right)_{i+\frac{1}{2}}^{n} \\
f_{1,1}\left(U_{1}\right)_{i+\frac{1}{2}}^{n}+f_{2,1}\left(U_{2}\right)_{i+\frac{1}{2}}^{n} \\
f_{1,2}\left(U_{1}\right)_{i+\frac{1}{2}}^{n}+f_{2,2}\left(U_{2}\right)_{i+\frac{1}{2}}^{n} \\
f_{1,3}\left(U_{1}\right)_{i+\frac{1}{2}}^{n}+f_{2,3}\left(U_{2}\right)_{i+\frac{1}{2}}^{n}
\end{array}\right)
$$

where $U_{k} \equiv\left(\rho_{k}, \rho_{k} u_{k}, \rho_{k} E_{k}\right)$ and $f_{k} \equiv\left(\rho_{k} u_{k}, \rho_{k} u_{k}^{2}+P_{k},\left(\rho_{k} E_{k}+P_{k}\right) u_{k}\right)$. Then, under the Hypothesis 2.1 and 3.1, the Eulerian numerical scheme (36) is consistent with (1) closed by (3)-(10), and is entropic under a CFL criterion.

Proof of Theorem 3.1. Let us define the numerical schemes

$$
\left\{\begin{array}{c}
U_{1, i}^{*}=U_{1, i}^{n}-\frac{\Delta t}{\Delta x_{i}}\left[f_{1}\left(U_{1}\right)_{i+\frac{1}{2}}^{n}-f_{1}\left(U_{1}\right)_{i-\frac{1}{2}}^{n}\right], \\
U_{2, i}^{*}=U_{2, i}^{n}-\frac{\Delta t}{\Delta x_{i}}\left[f_{2}\left(U_{2}\right)_{i+\frac{1}{2}}^{n}-f_{2}\left(U_{2}\right)_{i-\frac{1}{2}}^{n}\right]
\end{array}\right.
$$

which discretize (33) for $k \in\{1,2\}$.

Consistency: It is obvious by summing (38a) and (38b), and by using the point $i$ of the Property 3.1 .

Entropic property: Under the Hypothesis 2.1 and 3.1, we can write that

$$
\left(\rho_{k} s_{k}\right)_{i}^{*} \leq\left(\rho_{k} s_{k}\right)_{i}^{n}-\frac{\Delta t}{\Delta x_{i}}\left[g_{k}\left(U_{k}\right)_{i+\frac{1}{2}}^{n}-g_{k}\left(U_{k}\right)_{i-\frac{1}{2}}^{n}\right]
$$

under a CFL criterion for $k \in\{1,2\}$ where $g_{k}$ is the numerical entropic flux associated to the entropic scheme (38a) or (38b). By summing $\left(\rho_{1} s_{1}\right)_{i}^{*}$ with $\left(\rho_{2} s_{2}\right)_{i}^{*}$ and by noting that

$$
\rho_{k, i}^{*}=Y_{k, i}^{*} \rho_{i}^{*}=Y_{k, i}^{n+1} \rho_{i}^{n+1}=\rho_{k, i}^{n+1}
$$

because of the mixture relation (3c) and the relations (35), we obtain that

$$
\rho_{i}^{n+1}\left(Y_{1, i}^{n+1} s_{1, i}^{*}+Y_{2, i}^{n+1} s_{2, i}^{*}\right) \leq \rho_{i}^{n} s_{i}^{n}-\frac{\Delta t}{\Delta x_{i}}\left[g_{i+\frac{1}{2}}^{n}-g_{i-\frac{1}{2}}^{n}\right]
$$

where

$$
\left\{\begin{array}{l}
s=Y_{1} s_{1}+Y_{2} s_{2}, \\
g=g_{1}+g_{2} .
\end{array}\right.
$$

And, by applying the minimization principle (15)-(16), we deduce that the relaxation stage (35) closed with (12) to obtain $P_{k}^{n+1}$ and $T^{n+1}$ implies that

$$
s_{i}^{n+1} \leq Y_{1, i}^{n+1} s_{1, i}^{*}+Y_{2, i}^{n+1} s_{2, i}^{*}
$$

which concludes the proof. 


\subsubsection{For a fluid mixture in Lagrangian variables}

We now discretize the multicomponent Euler system written in Lagrangian variables

$$
\left\{\begin{array}{l}
\forall k \in\{1, \ldots, \bar{k}-1\}: \partial_{t} Y_{k}=0 \\
\partial_{t} \tau=\partial_{m} u \\
\partial_{t} u=-\partial_{m} P \\
\partial_{t} E=-\partial_{m}(P u)
\end{array}\right.
$$

now closed by (4)-(10).

\section{Hydrodynamic stage:}

The two numerical schemes solving the hydrodynamic stages (34) are given by

$$
\left\{\begin{array}{c}
U_{1, i}^{*}=U_{1, i}^{n}-\frac{\Delta t}{\Delta m_{1, i}^{n}}\left[f_{1}\left(U_{1}\right)_{i+\frac{1}{2}}^{n}-f_{1}\left(U_{1}\right)_{i-\frac{1}{2}}^{n}\right], \\
U_{2, i}^{*}=U_{2, i}^{n}-\frac{\Delta t}{\Delta m_{2, i}^{n}}\left[f_{2}\left(U_{2}\right)_{i+\frac{1}{2}}^{n}-f_{2}\left(U_{2}\right)_{i-\frac{1}{2}}^{n}\right]
\end{array}\right.
$$

with $\Delta m_{k, i}^{n}=\rho_{k, i}^{n} \Delta x_{i}^{n}$ and where $f_{1}=\left(f_{1,1}, f_{1,2}, f_{1,3}\right)$ and $f_{2}=\left(f_{2,1}, f_{2,2}, f_{2,3}\right)$ are the respective Lagrangian numerical fluxes. Moreover, the closure law (24b) imposes that

$$
\alpha_{k}^{*}=\alpha_{k}^{n}
$$

since the fluid mixture is initially at thermodynamic equilibrium (i.e. $u_{1}^{n}=u_{2}^{n}=U_{\text {int }}^{n}$ and $P_{1}^{n}=P_{2}^{n}=P_{\text {int }}^{n}$ ) and since the scheme is explicit.

Relaxation stage:

According to (35), we relaxe the mixture to the thermodynamic equilibrium by defining

$$
\left\{\begin{array}{l}
Y_{k, i}^{n+1}=Y_{k, i}^{*} \\
u_{i}^{n+1}=Y_{1, i}^{*} u_{1, i}^{*}+Y_{2, i}^{*} u_{2, i}^{*} \\
E_{i}^{n+1}=Y_{1, i}^{*} E_{1, i}^{*}+Y_{2, i}^{*} E_{2, i}^{*}
\end{array}\right.
$$

and we obtain the density $\rho_{i}^{n+1}$ with

$$
\frac{1}{\rho_{i}^{n+1}}=\frac{Y_{1, i}^{*}}{\rho_{1, i}^{*}}+\frac{Y_{2, i}^{*}}{\rho_{2, i}^{*}}
$$

by using the mixture law (4c). The quantities $T_{i}^{n+1}$ and $P_{i}^{n+1}$ are computed with (13).

To obtain the entropic results in Lagrangian variables, we have to verify a supplementary hypothesis relative to the numerical fluxes $f_{k}$ :

Hypothesis 3.2. We can modify the numerical flux $f_{k}=\left(f_{k, 1}, f_{k, 2}, f_{k, 3}\right)^{t}$ for each species $k$ such that

$$
f_{1,1}=f_{2,1}
$$

when the isothermal-isobaric-isovelocity laws (2)-(4b)-(10) are verified and such that the Hypothesis 3.1 is still verified. 
We have the following result:

Theorem 3.2. Under the Hypothesis 2.1 and 3.2, the Lagrangian numerical scheme (42)-(45) is consistent with (1) closed by (4)-(10) and is entropic under a CFL criterion. Moreover, it is conservative as soon as the fluxes $f_{k, 2}$ and $f_{k, 3}$ for each species $k$ verify

$$
\left\{\begin{array}{l}
f_{1,2}=f_{2,2}, \quad(a) \\
f_{1,3}=f_{2,3}
\end{array}\right.
$$

under the Hypothesis 3.2.

Proof of Theorem 3.2. The proof is similar to the proof of Theorem 3.1. We just have to underline that the equality (46) is crucial since it allows to write that the scheme $(42,43)$ implies that $Y_{1, i}^{*}=Y_{1, i}^{n}$ and $Y_{2, i}^{*}=Y_{2, i}^{n}$ which makes possible to use the minimization principle $(17,18)$ knowing the relations $(44,45)$.

Let us remark that the Hypothesis 3.2 coupled with (47) is very restrictive. Nevertheless, we will see in Section 3.3.2 that the set of such entropic schemes is not empty. At last, let us notice that a theorem similar to Theorem 3.2 could be obtained for a gas mixture.

\subsubsection{For a fluid mixture in Eulerian variables}

We explain in this section why, contrary to a gas mixture ( $c f$. Sect. 3.2.1), it seems impossible to prove that the relaxation scheme is entropic in Eulerian variables for a fluid mixture. We can already say that this impossibility is due to the mixture law (4c).

Indeed, in Eulerian variables, the hydrodynamic stage (33) for a fluid mixture is exactly the same than the hydrodynamic stage for a gas mixture; we just have also to solve the transport equation (24b) in order to know $\alpha_{k, i}^{*}$ and $Y_{k, i}^{*}$. And, under the Hypothesis 3.1, we obtain the inequalities (39). Nevertheless, the relaxation stage for a fluid mixture is different from the relaxation stage for a gas mixture since the mixture laws are different. And, it is easy to verify that the inequalities (39) do not imply the inequality (40) because of the mixture law $(4 \mathrm{c})$. As a consequence, we can not use the minimization principle $(17,18)$ to obtain the entropic result.

\subsection{Two examples of relaxation schemes}

We propose to present two examples of explicit finite volume schemes for the multicomponent Euler system (1) closed with (3)-(10) or with (4)-(10) which are already known and which can be interpreted as relaxation schemes. Then, it will be possible to immediately prove that these schemes are entropic under a CFL criterion by applying the Theorems 3.1 or 3.2 .

The first example is a kinetic scheme built for a gas mixture and proposed in [20]; the second one comes from [13] and was extended in [16] for a fluid mixture.

\subsubsection{For a gas mixture in Eulerian variables}

Let us suppose that each gas is ideal which means that each equation of state is given by (cf. [14] for example)

$$
\left\{\begin{aligned}
P_{k} & =\rho_{k} T_{k} / m_{k} \\
\varepsilon_{k} & =\mathcal{E}_{k}\left(T_{k}\right)
\end{aligned}\right.
$$

$\left(m_{k}\right.$ is the atomic mass of the species $k$ ), and let us suppose that each numerical flux $\left(f_{k, 1}, f_{k, 2}, f_{k, 3}\right)$ is defined by a mono-species kinetic scheme verifying the Hypothesis 3.1 as it is the case in [19] and [20].

Then, by applying the Theorem 3.1, we immediately obtain that the multicomponent kinetic scheme defined with $(36,37)$ is entropic under a CFL criterion: this result was previously proposed in Section 4.4 of [20] where it is proven entropic results in a more general context. 


\subsubsection{For a fluid mixture in Lagrangian variables}

Let us define the Lagrangian scheme for a fluid mixture

$$
\left\{\begin{aligned}
Y_{k, i}^{n+1} & =Y_{k, i}^{n}, \\
\tau_{i}^{n+1} & =\tau_{i}^{n}+\frac{\Delta t}{\Delta m_{i}}\left(u_{i+1 / 2}^{n}-u_{i-1 / 2}^{n}\right), \\
u_{i}^{n+1} & =u_{i}^{n}-\frac{\Delta t}{\Delta m_{i}}\left(P_{i+1 / 2}^{n}-P_{i-1 / 2}^{n}\right), \\
E_{i}^{n+1} & =E_{i}^{n}-\frac{\Delta t}{\Delta m_{i}}\left(P_{i+1 / 2}^{n} u_{i+1 / 2}^{n}-P_{i-1 / 2}^{n} u_{i-1 / 2}^{n}\right)
\end{aligned}\right.
$$

with $\Delta m_{i}=\rho_{i}^{0} \Delta x_{i}^{0}$ and with

$$
\left\{\begin{array}{l}
u_{i+1 / 2}=\frac{u_{i}+u_{i+1}}{2}-\frac{1}{2(\rho c)_{i+1 / 2}}\left(P_{i+1}-P_{i}\right) \\
P_{i+1 / 2}=\frac{P_{i}+P_{i+1}}{2}-\frac{(\rho c)_{i+1 / 2}}{2}\left(u_{i+1}-u_{i}\right)
\end{array}\right.
$$

And, to obtain $T^{n+1}$ and $P^{n+1}$, we solve the algebraic system (13).

The term $(\rho c)_{i+1 / 2}$ is strictly positive and is defined by

$$
(\rho c)_{i+1 / 2}=\sqrt{\frac{\max \left(\rho_{i} c_{i}^{2}, \rho_{i+1} c_{i+1}^{2}\right)}{\min \left(\frac{1}{\rho_{i}}, \frac{1}{\rho_{i+1}}\right)}}
$$

in [16] where $c$ is the sound speed of the fluid mixture (of course, we suppose that the Hypothesis 2.1 is verified: thus, because of the point $i i$ of Theorem 2.1, we can define $c$ and we have $c>0)$. Let us notice that $(\rho c)_{i+1 / 2}$ is proportional to the numerical viscosity of the scheme.

Let us now define the mono-species Lagrangian schemes for each species $k$ with (42) where the numerical flux $\left(f_{k, 1}, f_{k, 2}, f_{k, 3}\right)$ is defined by

$$
\left\{\begin{array}{l}
\left(f_{k, 1}\right)_{i+1 / 2}=u_{i+1 / 2} \\
\left(f_{k, 2}\right)_{i+1 / 2}=P_{i+1 / 2} \\
\left(f_{k, 3}\right)_{i+1 / 2}=P_{i+1 / 2} u_{i+1 / 2} .
\end{array}\right.
$$

In [13], it is proven that the mono-species numerical schemes (42a) or (42b) with (51) is entropic under a CFL criterion for any strictly positive choice of $(\rho c)_{i+1 / 2}$ (the choice of the formula giving $(\rho c)_{i+1 / 2}$ defines the value of the CFL constant). Then, the Hypothesis 3.2 and (47) are verified and we can rewrite the scheme (48) closed with (13) with the relaxation formalism (42)-(45). This implies, by applying the Theorem 3.2, that the Lagrangian scheme (48)-(50) closed with (13) is entropic under a CFL criterion.

\section{Chapman-Enskog expansion}

In order to formally prove that the relaxed system (20) is "well posed" when $\lambda_{u}, \lambda_{T}$ and, for a fluid mixture, $\lambda_{P}$ go to zero and that the relaxation procedure described in Section 3.2 is consistent with the multicomponent Euler system (1) closed with (3)-(10) or with (4)-(10), we compute a first order Chapman-Enskog expansion for a binary mixture.

In the case of a gas mixture, we will recover a binary Navier-Stokes system with the classical Fick and Newton laws, but with also the pressure diffusion and the thermal diffusion which are less known diffusion processes; in the case of a fluid mixture, we will also recover a binary Navier-Stokes system with the Fick and Newton laws, and with also two other diffusion laws: the first one is a density diffusion whose physical meaning is not clear, and the second one is a thermal diffusion similar to the previous one obtained for a gas mixture. 
The thermal diffusion in the case of a fluid mixture can be seen as a Soret effect. Indeed, the Soret effect is a diffusion effect in liquid mixtures which is equivalent to the thermal diffusion in gas mixtures. Let us notice that, contrary to the thermal diffusion in gas mixtures ( $c f$. [8]), it does not exist any theoretical derivation of the Soret effect (cf. [15], Chap. 8).

We will also underline that under the Hypothesis 2.1 and under the conditions (27) or (28), firstly, it is possible to compute the previous Chapman-Enskog expansion and, secondly, the coefficient of the Fick law has the good sign. Moreover, we will prove that the viscosity for a binary gas or fluid mixture is strictly positive when each species is ideal. We will also show that the thermal diffusion coefficient in gas mixture depends on the size of particles which is coherent with the Chapman and Cowling theory $(c f .[5,8]$ and $[15])$.

All these results will allow us to show similarities between this Chapman-Enskog expansion and the ChapmanEnskog expansion applied to classical Boltzmann equations ( $c f$. [8] for example): indeed, we will see that we can compute the first order Chapman-Enskog expansion of the relaxed multicomponent Euler system (20) when the Hypothesis 2.1 is verified and that it is also the case for the multi-species Boltzmann system when the micro-reversibility hypothesis applied to the microscopic cross sections is verified, hypothesis which is necessary to obtain a (kinetic) H-theorem and, thus, to prove the convergence of the distribution of each species $k$ to a Maxwellian distribution ( $c f$. Lem. 3.1 for the Euler system).

Let us notice that we suppose that the relaxation process has only one time scale: thus, we impose that $\lambda_{u}=\lambda_{T}=\lambda_{P} \equiv \lambda$ in $(20)$

\subsection{The first order Chapman-Enskog expansion}

The main results - whose proof are given in Appendix A - are the following:

Theorem 4.1. Under the Hypothesis 2.1 and under the conditions (27) or (28), it exists an unique $\left(J_{k}, \mu\right)$ such that the first order Chapman-Enskog expansion of binary multicomponent Euler system (20) closed with (21, 22) for a gas mixture or with (23, 24) for a fluid mixture is given by the multicomponent Navier-Stokes system

$$
\left\{\begin{array}{l}
\forall k: \partial_{t}\left(Y_{k} \rho\right)+\partial_{x}\left(Y_{k} \rho u\right)=\partial_{x}\left(\lambda J_{k}\right) \\
\partial_{t}(\rho u)+\partial_{x}\left(\rho u^{2}+P\right)=\partial_{x}\left(\lambda \mu \partial_{x} u\right) \\
\partial_{t}(\rho E)+\partial_{x}[(\rho E+P) u]=\partial_{x}\left[\lambda\left(J_{1} h_{1}+J_{2} h_{2}\right)\right]+\partial_{x}\left(\lambda \mu u \partial_{x} u\right)
\end{array}\right.
$$

where $h_{k}=\varepsilon_{k}+P_{k} / \rho_{k}$ is the specific enthalpy of the species $k$, system which is closed with (3)-(10) for a gas mixture or with (4)-(10) for a fluid mixture.

Let us notice that $\mu$ can be assimilated to the classical viscosity of the Newton law.

And, it is possible to explicit the formula giving the flux $J_{k}$ :

Proposition 4.1. Under the Hypothesis 2.1 and by defining

$$
\Theta_{12}\left(T, P_{1}, P_{2}\right)=Y_{1} \frac{\partial \tau_{1}}{\partial P_{1}}\left(T, P_{1}\right)+Y_{2} \frac{\partial \tau_{2}}{\partial P_{2}}\left(T, P_{2}\right)
$$

we have:

i) For a gas mixture, the flux $J_{k}$ in (52) is defined by

$$
J_{k}=\chi \partial_{x} Y_{k}+\beta_{k} \partial_{x} P+\gamma_{k} \partial_{x} T
$$


where

$$
\left\{\begin{array}{l}
\chi=-\rho Y_{1} Y_{2} \frac{\tau_{1}+\tau_{2}}{\Theta_{12}}, \\
\beta_{1}=\rho Y_{1} Y_{2}\left(\frac{Y_{2} \frac{\partial \tau_{2}}{\partial P_{2}}\left(T, P_{2}\right)}{\Theta_{12}}-Y_{1}\right) \in[-1,1], \\
\gamma_{1}=\rho Y_{1} Y_{2} \frac{Y_{2} \frac{\partial \tau_{2}}{\partial T_{2}}\left(T, P_{2}\right)-Y_{1} \frac{\partial \tau_{1}}{\partial T_{1}}\left(T, P_{1}\right)}{\Theta_{12}}
\end{array}\right.
$$

with $\Theta_{12}=\Theta_{12}\left(T, P_{1}, P_{2}\right)$.

ii) For a fluid mixture, the flux $J_{k}$ in (52) is defined by

$$
J_{k}=\widetilde{\chi} \partial_{x} Y_{k}+\widetilde{\beta_{k}} \partial_{x} \rho+\widetilde{\gamma_{k}} \partial_{x} T
$$

where

with $\Theta_{12}=\Theta_{12}(T, P, P)$.

$$
\left\{\begin{array}{l}
\widetilde{\chi}=-\left(\rho Y_{1} Y_{2}\right)^{2} \frac{\left(\tau_{1}-\tau_{2}\right)^{2}}{\Theta_{12}} \\
\widetilde{\beta_{1}}=\left(Y_{1} Y_{2}\right)^{2} \frac{\tau_{2}-\tau_{1}}{\Theta_{12}}, \\
\widetilde{\gamma_{1}}=\left(\rho Y_{1} Y_{2}\right)^{2}\left(\tau_{2}-\tau_{1}\right) \frac{Y_{1} \frac{\partial \tau_{1}}{\partial T_{1}}(T, P)+Y_{2} \frac{\partial \tau_{2}}{\partial T_{2}}(T, P)}{\Theta_{12}}
\end{array}\right.
$$

And we have $\beta_{2}=-\beta_{1}, \gamma_{2}=-\gamma_{1}$ and $\widetilde{\beta_{2}}=-\widetilde{\beta_{1}}, \widetilde{\gamma_{1}}=-\widetilde{\gamma_{2}}$.

The fluxes $\chi \partial_{x} Y_{k}$ and $\tilde{\chi} \partial_{x} Y_{k}$ in (54) and (56) model the classical Fick law. The fluxes $\beta_{k} \partial_{x} P$ and $\gamma_{k} \partial_{x} T$ in (54) model for a gas mixture the diffusion through the respective pressure and thermal diffusions which are less known than the Fick law (cf. [8] and [5]).

The value of $\gamma_{1}$ in (55) is formally similar to the formula

$$
\gamma_{1}=\rho Y_{1} Y_{2} \frac{Y_{2} \mathcal{S}_{2}-Y_{1} \mathcal{S}_{1}}{\mathcal{S}_{12}}
$$

given in [8] ( $c f$. formula $(9.83,1)$ p. 165) where $\mathcal{S}_{k}$ and $\mathcal{S}_{12}$ depend on the type of microscopic interaction between the particles 1 and 2 .

For a fluid mixture, we also find two terms in $(56)$ - namely $\widetilde{\beta_{k}} \partial_{x} \rho$ and $\widetilde{\gamma_{k}} \partial_{x} T$ - which are similar to the previous pressure and thermal diffusions for a gas mixture.

Concerning the term $\widetilde{\beta_{k}} \partial_{x} \rho$, we can say that the density $\rho=\alpha_{1} \rho_{1}+\alpha_{2} \rho_{2}$ in $J_{k}$ for a fluid mixture plays the role of the pressure $P=\alpha_{1} P_{1}+\alpha_{2} P_{2}$ in $J_{k}$ for a gas mixture (we recall that $\alpha_{1}=\alpha_{2}=1$ in a gas mixture). But, its physical meaning is not obvious.

Nevertheless, the term $\widetilde{\gamma_{k}} \partial_{x} T$ has a physical meaning: indeed, it can be assimilated to a Soret effect which defines the thermal diffusion in a liquid mixture (cf. [15], Chap. 8).

Let us notice that there are no Fourier law and no Dufour law in the energy equation of (52): this shows that the relaxed system (20) does not take into account all the physics. Let us recall that the Dufour law (also called diffusion thermo-effect in [8]) models the creation of a temperature gradient through a mass fraction gradient and does not have to be confounded with the energy flux $\lambda \sum_{k} J_{k} h_{k}$ which is directly due to the interdiffusion between species. In other words, the Dufour effect is the "opposite" of the thermal diffusion or Soret effect. 
Let us remark that the thermal diffusion and Dufour effect are known to be very tiny for common temperature gradients (these diffusion phenomena are taken into account in combustion for example where the temperature gradients are very important).

We can deduce from the previous proposition that:

Corollary 4.2. Under the Hypothesis 2.1, the Fick coefficients $\chi$ and $\tilde{\chi}$ are positive.

Unfortunately, it seems a priori more difficult to obtain a simple formula giving the viscosity $\mu$ which shows that $\mu>0$ under the Hypothesis 2.1 for any equation of state although this last result should be true. Nevertheless, in the following subsection, we will show that this viscosity is strictly positive when all the species are ideal.

Proof of corollary 4.2. We just have to prove that $\Theta_{12}<0$ under the Hypothesis 2.1. This inequality is deduced from the point $i$ of the Lemma B.1 in Appendix B.

\subsection{Computation of the diffusion coefficients for ideal species}

We now particularize the results of the Theorem 4.1 and of the Proposition 4.1:

\section{Computation of the viscosity for a binary mixture when the species are ideal}

Let us now suppose that each equation of state $\varepsilon_{k}\left(\tau_{k}, P_{k}\right)$ is given by

$$
\left\{\begin{array}{l}
P_{k}=\rho_{k} T_{k} / m_{k}, \\
\varepsilon_{k}=\mathcal{E}_{k}\left(T_{k}\right)
\end{array}\right.
$$

where $m_{k}$ is the atomic mass of the species $k$. Thus, each species $k$ is ideal and we can establish the following result (the proof is in Appendix C):

Lemma 4.1. When the equation of state $\varepsilon_{1}\left(\tau_{1}, P_{1}\right)$ and $\varepsilon_{2}\left(\tau_{2}, P_{2}\right)$ are defined by (58), the Newton coefficient $\mu$ verifies

$$
\mu=\frac{T\left(Y_{1} Y_{2} \rho\right)^{2}}{m_{1} m_{2}}\left(\frac{\sqrt{\frac{m_{1}}{m_{2}}} \mathcal{E}_{1}(T)^{\prime}-\sqrt{\frac{m_{2}}{m_{1}}} \mathcal{E}_{2}(T)^{\prime}}{Y_{1} \mathcal{E}_{1}(T)^{\prime}+Y_{2} \mathcal{E}_{2}(T)^{\prime}}\right)^{2}>0
$$

for a gas or fluid mixture.

\section{Computation of the flux $J_{k}$ for a binary gas mixture when the species are ideal}

Lemma 4.2. When the equation of state $\varepsilon_{1}\left(\tau_{1}, P_{1}\right)$ and $\varepsilon_{2}\left(\tau_{2}, P_{2}\right)$ are defined by (58) in a gas mixture, the thermal diffusion does not exist (i.e. $\gamma_{k}=0$ ) and the flux $J_{k}$ given by (54) can be rewritten with

$$
J_{k}=D_{12}\left[\partial_{x} c_{k}+\left(1-\frac{m_{k}}{m}\right) c_{k} \partial_{x} \log P\right]
$$

where $D_{12}=D_{21}>0$ knowing that $\frac{1}{m} \equiv \frac{Y_{1}}{m_{1}}+\frac{Y_{2}}{m_{2}}$ and $c_{k} \equiv Y_{k} m / m_{k}$ which is the concentration of the species $k$.

Let us remark that the flux (60) is classical in isotopic separation. Moreover, it is known that for ideal gases, the thermal diffusion effect is almost equal to zero compared to the Fick and pressure diffusion effects. 
Computation of the thermal diffusion in a binary gas mixture when only one of the species is ideal

Now, $\varepsilon_{1}\left(\tau_{1}, P_{1}\right)$ and $\varepsilon_{2}\left(\tau_{2}, P_{2}\right)$ are respectively given by (58) and by

$$
\left\{\begin{array}{l}
P_{k}=\frac{T_{k} / m_{k}}{\tau_{k}-b_{k}}, \\
\varepsilon_{k}=\mathcal{E}_{k}\left(T_{k}\right)
\end{array}\right.
$$

where the strictly positive constant $b_{k}$ is called covolume. This covolume takes into account in a rough way the fact that there is a compressibility limit for the gas 2 because of the size of the atoms or molecules constituting this gas (here, the law (61) imposes that $\rho_{2}<b_{2}^{-1}$ ). In this case, we obtain the following lemma:

Lemma 4.3. When the equations of state $\varepsilon_{1}\left(\tau_{1}, P_{1}\right)$ and $\varepsilon_{2}\left(\tau_{2}, P_{2}\right)$ are respectively defined by (58) and (61), it exists $\Gamma_{12}>0$ such that

$$
\gamma_{1}=\rho Y_{1} Y_{2} \Gamma_{12} b_{2}>0 .
$$

We deduce of this lemma that the thermal diffusion depends on the size of the particles of each species which is coherent with the Chapman and Cowling theory ( $c f .[8])$. Let us notice that the positive sign of $\gamma_{1}$ in (62) implies that the more the size of the particle of type 2 is important, the more they diffuse to the hot area: in general, the Chapman and Cowling theory shows that $\gamma_{1}<0$; but, for particular microscopic interaction models as the Lennard-Jones model, $\gamma_{1}$ can be positive (see Figs. 9 and 10 in Sect. 2.7 of [15]).

\subsection{Comparison with the Chapman-Enskog expansion applied to Boltzmann equations}

An important remark is that we are able to compute the first order perturbations of the Chapman-Enskog expansion for the relaxed Euler system (20) only under the thermodynamic Hypothesis 2.1 (and under the conditions (27) for a gas mixture or (28) for a fluid mixture).

Similarly, for the multi-species Boltzmann system ( $c f$. [22]), we are able to compute the first order perturbations of the Chapman-Enskog expansion when the micro-reversibility hypothesis is verified, hypothesis which imposes that the microscopic cross sections of the Boltzmann operator have a particular form (see Sect. 3 and the remark at the end of Sect. 6 in [11] for example). Indeed, a mono-species or multi-species Boltzmann operator admits only one equilibrium distribution ( $c f$. the point $i$ of the Th. 2.1 for the Euler system) - namely a Maxwellian distribution in the classical cases - and a H-theorem ( $c f$. the Lem. 3.1 for the Euler system) when the micro-reversibility hypothesis is fulfilled.

More precisely, we have seen in the proof of Theorem 4.1 ( $c f$. Prop. A.1 in Appendix A) that, under the Hypothesis 2.1, the first order perturbation $X$ of the Chapman-Enskog expansion is solution of a linear matricial system of the type $M \cdot X=B$; in the Boltzmann case, we could prove that the corresponding perturbation $X$ is solution of a linear system of the form $L(X)=g$ where $L$ is a linear integral operator and where $g \in \operatorname{Ker}(L)^{\perp}$ when the micro-reversibility hypothesis is verified. And, these two linear problems have an unique solution because:

- for the relaxed multicomponent Euler system: $\operatorname{det}(M)>0(c f$. Appendix A);

- for the multi-species Boltzmann operator: we can apply the Fredholm alternative on the linear operator $L$.

Thus, we can say that the sentence

each macroscopic equation of state in the relaxed multicomponent Euler system admits a convex entropy is qualitatively equivalent to the sentence

each microscopic cross section in the multi-species Boltzmann system verifies the micro-reversibility property. 
TABLE 1.

\begin{tabular}{|c|c|c|}
\hline & Relaxed Euler system & Boltzmann system \\
\hline$\overline{\text { Perturbation }}$ & $\begin{array}{l}X=\left(P_{k 1}, T_{k 1}\right)(t, x) \\
(c f . \text { Appendix A) }\end{array}$ & $\begin{array}{l}X=\left(X_{k}\right)(t, x, v) \\
(v \text { is the microscopic velocity) }\end{array}$ \\
\hline Condition & $\begin{array}{l}\exists s_{k} \text { strict. conv., } 27 \text { or } 28 \text { is verified } \\
\quad \Downarrow \\
\exists \text { a H-theorem }\end{array}$ & $\begin{array}{l}\text { micro-reversibility hypothesis } \\
\quad \Downarrow \\
\exists \text { a H-theorem }\end{array}$ \\
\hline Linear Equation & $\begin{array}{l}\exists(M, B) \text { such that } \\
M \cdot X=B(t, x)\end{array}$ & $\begin{array}{l}\exists\left(L, g \in \operatorname{Ker}(L)^{\perp}\right) \text { such that } \\
L(X)=g(t, x, v)\end{array}$ \\
\hline $\begin{array}{l}\text { Existence } \\
\text { and unicity }\end{array}$ & $\begin{array}{l}\operatorname{det}(M)>0 \\
\text { (because } s_{k} \text { is strict. conv.) }\end{array}$ & Fredholm alternative \\
\hline $\begin{array}{l}\text { Transport coefs. } \\
\text { for a binary ideal } \\
\text { gas mixture }\end{array}$ & $\begin{array}{l}\text { Fick }>0 \\
\text { Pressure diffusion } \neq 0 \\
\text { Newton }>0 \\
\text { No Fourier } \\
\text { No thermal diff. and no Dufour effect }\end{array}$ & $\begin{array}{l}\text { Fick }>0 \\
\text { Pressure diffusion } \neq 0 \\
\text { Newton }>0 \\
\text { Fourier }>0 \\
\text { Thermal diff. and Dufour effect } \\
\text { can exist but are often neglected }\end{array}$ \\
\hline
\end{tabular}

We summarize the previous remarks in the Table 1.

\section{Conclusion}

After having defined a gas mixture as an intimate mixture of gases and a fluid mixture as an immiscible mixture of fluids (or separated phase mixture), we have extended the relaxation schemes initially proposed in [7] for the mono-species Euler system to the multicomponent Euler system describing an isothermal-isovelocity gas mixture or an isothermal-isobaric-isovelocity fluid mixture by solving with a splitting technique a relaxed multicomponent Euler system. And, with the help of a Gibbs lemma, we have proven entropic results on these relaxation schemes in Eulerian variables for a gas mixture and in Lagrangian variables for a fluid mixture. These results allowed us to prove that a multicomponent kinetic Eulerian scheme is entropic under a CFL criterion (result already proven in [20]) and that the multicomponent version of the Lagrangian scheme described in [13] and used in [16] is also entropic under a CFL criterion. It is important to recall that these relaxation schemes in the case of fluid mixtures are not dedicated to simulations of interfaces problems where the fluid mixture is numerical and located to material interfaces, but to simulations of physical fluid mixtures at thermodynamic equilibrium (as in the case of gas mixtures) and at scales where it is impossible to define any material interface between each fluid.

After having proven these entropic results, we have performed a first order Chapman-Enskog expansion on the previous relaxed system for a binary mixture. This has allowed us to obtain a binary Navier-Stokes system including the Fick and Newton laws for a gas and fluid mixture. We have also found the pressure and thermal diffusions for a gas mixture and, for a fluid mixture, a density diffusion - whose physical meaning is not clear - and a thermal diffusion which can be assimilated to a Soret effect. Moreover, for particular equations of state, we have recovered some physical results concerning the sign of the diffusion constants. At last, we have underlined similarities between this Chapman-Enskog expansion and the Chapman-Enskog expansion applied to classical Boltzmann equations.

A next work could be to find a good projection stage to obtain an entropic Eulerian scheme for a fluid mixture by using the Lagrange + projection technique described in [16] and by using the entropic Lagrangian scheme proposed in this paper. At last, it should be interesting to specify the results on the positivity of the viscosity in the Chapman-Enskog expansion for any equation of state and for any number of species in the mixture. 


\section{Appendix A. Proof ot Theorem 4.1 And of Proposition 4.1}

\section{A.1. Proof of Theorem 4.1}

Let us suppose that at any point $(t, x)$, we know $\rho_{k} \equiv 1 / \tau_{k}, \alpha_{k}, u_{k}$ and $E_{k}$ with $k \in\{1,2\}$. Then, we know $\rho \equiv \alpha_{1} \rho_{1}+\alpha_{2} \rho_{2}, Y_{k} \equiv \alpha_{k} \rho_{k} / \rho, E \equiv Y_{1} E_{1}+Y_{2} E_{2}, u \equiv Y_{1} u_{1}+Y_{2} u_{2}, \varepsilon_{k} \equiv E_{k}-\frac{u_{k}^{2}}{2}$ and the unique $\left(T_{k}, P_{k}\right)$ such that $\tau_{k}\left(T_{k}, P_{k}\right)=\tau_{k}$ and $\varepsilon_{k}\left(T_{k}, P_{k}\right)=\varepsilon_{k}$. Moreover, we can define the associated thermodynamic equilibrium with

$$
\left\{\begin{array}{l}
\alpha_{k}^{\mathrm{eq}}=\alpha_{k} \equiv 1 \\
Y_{1} \tau_{1}\left(T^{\mathrm{eq}}, P_{1}^{\mathrm{eq}}\right)=\tau \\
Y_{2} \tau_{2}\left(T^{\mathrm{eq}}, P_{2}^{\mathrm{eq}}\right)=\tau \\
Y_{1} \varepsilon_{1}\left(T^{\mathrm{eq}}, P_{1}^{\mathrm{eq}}\right)+Y_{2} \varepsilon_{2}\left(T^{\mathrm{eq}}, P_{2}^{\mathrm{eq}}\right)=E-\frac{u^{2}}{2} \equiv \varepsilon^{\mathrm{eq}} \\
P^{\mathrm{eq}} \equiv P_{1}^{\mathrm{eq}}+P_{2}^{\mathrm{eq}}
\end{array}\right.
$$

for a gas mixture, and with

$$
\left\{\begin{array}{l}
\alpha_{1}^{\mathrm{eq}}+\alpha_{2}^{\mathrm{eq}}=\alpha_{1}+\alpha_{2} \equiv 1 \\
Y_{1} \tau_{1}\left(T^{\mathrm{eq}}, P_{1}^{\mathrm{eq}}\right)+Y_{2} \tau_{2}\left(T^{\mathrm{eq}}, P_{2}^{\mathrm{eq}}\right)=\tau \\
Y_{1} \varepsilon_{1}\left(T^{\mathrm{eq}}, P_{1}^{\mathrm{eq}}\right)+Y_{2} \varepsilon_{2}\left(T^{\mathrm{eq}}, P_{2}^{\mathrm{eq}}\right)=E-\frac{u^{2}}{2} \equiv \varepsilon^{\mathrm{eq}} \\
P^{\mathrm{eq}} \equiv P_{1}^{\mathrm{eq}}=P_{2}^{\mathrm{eq}}
\end{array}\right.
$$

for a fluid mixture where the subscript eq means equilibrium (we recall that this equilibrium is unique: $c f$. Th. 2.1, point $i))$. This allows to define $\tau_{k}^{\mathrm{eq}} \equiv \tau_{k}\left(T^{\mathrm{eq}}, P_{k}^{\mathrm{eq}}\right)$ and $\varepsilon_{k}^{\mathrm{eq}} \equiv \varepsilon_{k}\left(T^{\mathrm{eq}}, P_{k}^{\mathrm{eq}}\right)$. Let us notice that $Y_{k}^{\mathrm{eq}}=Y_{k}$, $\rho^{\mathrm{eq}}=\rho, \alpha_{k}^{\mathrm{eq}} \rho_{k}^{\mathrm{eq}}=\alpha_{k} \rho_{k}, u^{\mathrm{eq}}=u$ and $E^{\mathrm{eq}}=E$.

We will prove that this thermodynamic equilibrium is solution of (52) when $\lambda$ goes to zero. The first step is to suppose that the solution of binary multicomponent Euler system (20) can be expanded in the following way:

$$
\left(\begin{array}{c}
\alpha_{k} \\
u_{k} \\
P_{k} \\
T_{k} \\
\tau_{k} \\
\varepsilon_{k}
\end{array}\right)=\left(\begin{array}{c}
\alpha_{k}^{\mathrm{eq}} \\
u^{\mathrm{eq}} \\
P_{k}^{\mathrm{eq}} \\
T^{\mathrm{eq}} \\
\tau_{k}^{\mathrm{eq}} \\
\varepsilon_{k}^{\mathrm{eq}}
\end{array}\right)+\lambda\left(\begin{array}{c}
\alpha_{k 1} \\
u_{k 1} \\
P_{k 1} \\
T_{k 1} \\
\tau_{k 1} \\
\varepsilon_{k 1}
\end{array}\right)+\mathcal{O}\left(\lambda^{2}\right)
$$

We have the immediate property which is a consequence of (63), (64) and (65):

\section{Property A.1.}

Moreover, for a gas mixture

$$
\begin{cases}Y_{1} u_{11}+Y_{2} u_{21}=0 & (a) \\ Y_{1} \varepsilon_{11}+Y_{2} \varepsilon_{21}=0 & (b)\end{cases}
$$

$$
\alpha_{k 1}=0 \quad \text { and } \quad \tau_{k 1}=0 \text {, }
$$

and for a fluid mixture

$$
\left\{\begin{array}{l}
\alpha_{11}+\alpha_{21}=0, \\
Y_{1} \tau_{11}+Y_{2} \tau_{21}=0 .
\end{array}\right.
$$




\section{A.1.1. Continuity equations}

We prove the following lemma:

\section{Lemma A.1.}

i)

$$
\partial_{t}\left(Y_{1} \rho\right)+\partial_{x}\left(Y_{1} \rho u^{\mathrm{eq}}\right)=\partial_{x}\left(\lambda J_{1}\right)+\mathcal{O}\left(\lambda^{2}\right)
$$

with $J_{1} \equiv-\rho Y_{1} u_{11}$.

ii) For a gas mixture:

$$
u_{11}=-Y_{2}\left(Y_{2} \partial_{x} P_{1}^{\mathrm{eq}}-Y_{1} \partial_{x} P_{2}^{\mathrm{eq}}\right) .
$$

iii) For a fluid mixture:

$$
u_{11}=-Y_{1} Y_{2}\left(\frac{\tau_{1}^{\mathrm{eq}}}{\tau}-1\right) \partial_{x} P^{\mathrm{eq}}
$$

Proof of Lemma A.1. The point $i$ is deduced from (20a) and from the expansion (65). Moreover, the equations (20a) and (20b), the expansion (65) and the property (66a) imply that

$$
\partial_{t} u^{\mathrm{eq}}+u^{\mathrm{eq}} \partial_{x} u^{\mathrm{eq}}+\tau_{1}^{\mathrm{eq}} \partial_{x} P_{1}^{\mathrm{eq}}=-\frac{u_{11}}{Y_{1} Y_{2} \rho}+\mathcal{O}(\lambda)
$$

By summing (20b) and (20b') and by using again the expansion (65), we also have

$$
\partial_{t} u^{\mathrm{eq}}+u^{\mathrm{eq}} \partial_{x} u^{\mathrm{eq}}=-\tau \partial_{x} P^{\mathrm{eq}}+\mathcal{O}(\lambda)
$$

These two last equalities show that

$$
u_{11}=-Y_{1} Y_{2}\left(\frac{\tau_{1}^{\mathrm{eq}}}{\tau} \partial_{x} P_{1}^{\mathrm{eq}}-\partial_{x} P^{\mathrm{eq}}\right)
$$

We conclude the proof by using the relations $(3 \mathrm{~b}, \mathrm{c})$ or $(4 \mathrm{~b})$.

\section{A.1.2. Momentum equation}

As for the continuity equations, we easily obtain that

$$
\partial_{t}\left(\rho u^{\mathrm{eq}}\right)+\partial_{x}\left[\rho\left(u^{\mathrm{eq}}\right)^{2}+P^{\mathrm{eq}}\right]=-\partial_{x}\left[\lambda\left(\alpha_{1}^{\mathrm{eq}} P_{11}+\alpha_{2}^{\mathrm{eq}} P_{21}\right)\right]+\mathcal{O}\left(\lambda^{2}\right) .
$$

Then, we have to evaluate $\alpha_{1}^{\mathrm{eq}} P_{11}+\alpha_{2}^{\mathrm{eq}} P_{21}$. We have the following proposition:

Proposition A.1. Under the Hypothesis 2.1 and under the conditions (27) or (28), it exists a matrix $M_{\kappa}$ and two functions $\mathcal{A}(T, P)$ and $\mathcal{B}(T, P)$ which depend on the equations of state of each species such that the first order perturbations $P_{k 1}$ and $T_{k 1}$ are solution of linear system

$$
M_{\kappa} \cdot\left(\begin{array}{c}
P_{11} \\
P_{12} \\
T_{11} \\
T_{21}
\end{array}\right)=\left(\begin{array}{c}
0 \\
0 \\
\kappa Y_{1} Y_{2} \mathcal{B}^{\mathrm{eq}} \cdot \partial_{x} u^{\mathrm{eq}} \\
Y_{1} Y_{2} \mathcal{A}^{\mathrm{eq}} \cdot \partial_{x} u^{\mathrm{eq}}
\end{array}\right)
$$


with $\kappa=0$ for a gas mixture and $\kappa=1$ for a fluid mixture, and with $\mathcal{A}^{\mathrm{eq}} \equiv \mathcal{A}\left(T^{\mathrm{eq}}, P^{\mathrm{eq}}\right)$ and $\mathcal{B}^{\mathrm{eq}} \equiv \mathcal{B}\left(T^{\mathrm{eq}}, P^{\mathrm{eq}}\right)$. And, we have

$$
M_{\kappa=0}=\left(\begin{array}{cccc}
Y_{1} \frac{\partial \varepsilon_{1}}{\partial P_{1}} & Y_{2} \frac{\partial \varepsilon_{2}}{\partial P_{2}} & Y_{1} \frac{\partial \varepsilon_{1}}{\partial T_{1}} & Y_{2} \frac{\partial \varepsilon_{2}}{\partial T_{2}} \\
\frac{\partial \tau_{1}}{\partial P_{1}} & 0 & \frac{\partial \tau_{1}}{\partial T_{1}} & 0 \\
0 & \frac{\partial \tau_{2}}{\partial P_{2}} & 0 & \frac{\partial \tau_{2}}{\partial T_{2}} \\
0 & 0 & -1 & 1
\end{array}\right)
$$

and

$$
M_{\kappa=1}=\left(\begin{array}{cccc}
Y_{1} \frac{\partial \varepsilon_{1}}{\partial P_{1}} & Y_{2} \frac{\partial \varepsilon_{2}}{\partial P_{2}} & Y_{1} \frac{\partial \varepsilon_{1}}{\partial T_{1}} & Y_{2} \frac{\partial \varepsilon_{2}}{\partial T_{2}} \\
Y_{1} \frac{\partial \tau_{1}}{\partial P_{1}} & Y_{2} \frac{\partial \tau_{2}}{\partial P_{2}} & Y_{1} \frac{\partial \tau_{1}}{\partial T_{1}} & Y_{2} \frac{\partial \tau_{2}}{\partial T_{2}} \\
1 & -1 & 0 & 0 \\
0 & 0 & -1 & 1
\end{array}\right) .
$$

Moreover, $\operatorname{det}\left(M_{\kappa}\right)>0$ which allows to easily write that it exists an unique function $\mu(T, P)$ depending on the equations of state and of the type of mixture such that

$$
\alpha_{1}^{\mathrm{eq}} P_{11}+\alpha_{2}^{\mathrm{eq}} P_{21}=-\mu^{\mathrm{eq}} \cdot \partial_{x} u^{\mathrm{eq}} .
$$

Let us notice that the conditions (27) or (28) imply that $U_{\text {int }}=u^{\text {eq }}+\mathcal{O}(\lambda)$ for a gas or fluid mixture and that $P_{\text {int }}=P^{\mathrm{eq}}+\mathcal{O}(\lambda)$ for a fluid mixture: this property is necessary to obtain $\mathcal{A}$ and $\mathcal{B}$ and, thus, to obtain the correct first order perturbations. This remark coupled with the Lemma 3.1 (H-theorem) links clearly the existence of the diffusion processes in the spatially non homogeneous case with the existence of a convergence toward a thermodynamic equilibrium minimizing the mixture entropy in the spatially homogeneous case.

Corollary A.1. The functions $\mathcal{A}$ and $\mathcal{B}$ are defined with

$$
\mathcal{A}=T\left[-\frac{\partial\left(s_{1}-s_{2}\right)}{\partial \tau}+P \frac{\partial\left(s_{1}-s_{2}\right)}{\partial \varepsilon}\right]
$$

and

$$
\mathcal{B}=\frac{\partial\left(\tau_{1}-\tau_{2}\right)}{\partial \tau}-\frac{\tau_{1}-\tau_{2}}{\tau}-P \frac{\partial\left(\tau_{1}-\tau_{2}\right)}{\partial \varepsilon}
$$

Proof of Proposition A.1.

Computation of the first line of $M_{\kappa}$ and of the second line of $M_{\kappa=1}$ : A Taylor expansion of $\varepsilon_{k}$ around the equilibrium gives

$$
\varepsilon_{k}\left(P_{k}, T_{k}\right)=\varepsilon_{k}^{\mathrm{eq}}+\lambda\left(P_{k 1} \frac{\partial \varepsilon_{k}^{\mathrm{eq}}}{\partial P_{k}}+T_{k 1} \frac{\partial \varepsilon_{k}^{\mathrm{eq}}}{\partial T_{k}}\right)+\mathcal{O}\left(\lambda^{2}\right)
$$

which shows that $\varepsilon_{k 1}=P_{k 1} \frac{\partial \varepsilon_{p_{k}}^{\mathrm{eq}}}{\partial P_{k}}+T_{k 1} \frac{\partial \varepsilon_{k}^{\mathrm{eq}}}{\partial T_{k}}$. We conclude by using the property (66b). And, for a fluid mixture, the relations $(66 \mathrm{~b})$ and $(68 \mathrm{~b})$ show that $\left(\varepsilon_{k}, \varepsilon_{k 1}\right)$ and $\left(\tau_{k}, \tau_{k 1}\right)$ play the same rule from an algebraic point of view: then, we immediately obtain the second line of $M_{\kappa=1}$.

Computation of the second and third lines of $M_{\kappa=0}$ : We apply the previous technique by taking into account that for a gas mixture, we have $Y_{k} \tau_{k}=\tau$ and $\tau_{k 1}=0$ (cf. Property A.1). 
Computation of the fourth line of $M_{\kappa}$ and computation of $\mathcal{A}$ : Since $s_{1}$ is the thermodynamic entropy of the species 1 , we easily have that

$$
-T^{\mathrm{eq}} Y_{1} \rho\left(\partial_{t} s_{1}^{\mathrm{eq}}+u^{\mathrm{eq}} \partial_{x} s_{1}^{\mathrm{eq}}\right) \equiv-T^{\mathrm{eq}} Y_{1} \rho D_{t} s_{1}^{\mathrm{eq}}=\left(T_{21}-T_{11}\right)+\mathcal{O}(\lambda)
$$

under the conditions (27) or (28) and by using the closure law (24b) for a fluid mixture. By using the point $i$ of Theorem 2.1, we can write that $s_{k}^{\mathrm{eq}}=s_{k}\left(\tau, \varepsilon^{\mathrm{eq}}\right)$. Then, $D_{t} s_{1}^{\mathrm{eq}}=\frac{\partial s_{1}^{\mathrm{eq}}}{\partial \varepsilon} D_{t} \varepsilon^{\mathrm{eq}}+\frac{\partial s_{1}^{\mathrm{eq}}}{\partial \tau} D_{t} \tau$. And since $D_{t} \varepsilon^{\mathrm{eq}}=$ $-\tau P^{\mathrm{eq}} \partial_{x} u^{\mathrm{eq}}+\mathcal{O}(\lambda)$ and $D_{t} \tau=\tau \partial_{x} u^{\mathrm{eq}}+\mathcal{O}(\lambda)$, we obtain that $T_{21}-T_{11}=T Y_{1}\left(P \frac{\partial s_{1}^{\mathrm{eq}}}{\partial \varepsilon}-\frac{\partial s_{1}^{\mathrm{eq}}}{\partial \tau}\right) \partial_{x} u^{\mathrm{eq}}$ which allows to easily obtain the function $\mathcal{A}$ by symmetry with the entropy $s_{2}^{\text {eq }}$.

Computation of the third line of $M_{\kappa=1}$ and computation of $\mathcal{B}$ : The equation (25a) and the expansion (65) allow to write that

$$
D_{t} \tau_{1}^{\mathrm{eq}}=\tau^{\mathrm{eq}} \partial_{x} u^{\mathrm{eq}}+\tau_{1}^{\mathrm{eq}}\left(P_{11}-P_{21}\right)+\mathcal{O}(\lambda)
$$

as soon as the interfacial velocity $U_{\text {int }}$ verifies (28). Then, we continue the proof by noting that $D_{t} \tau=\tau \partial_{x} u^{\text {eq }}+$ $\mathcal{O}(\lambda)$ and by applying the previous technique used to evaluate $\mathcal{A}$.

Computation of $\operatorname{det}\left(M_{\kappa}\right)$ : Algebraic calculus show that

$$
\operatorname{det}\left(M_{\kappa=0}\right)=-Y_{1} \frac{\partial \tau_{2}}{\partial P_{2}}\left(T, P_{2}\right)\left(\frac{\partial \varepsilon_{1}}{\partial P_{1}} \frac{\partial \tau_{1}}{\partial T_{1}}-\frac{\partial \varepsilon_{1}}{\partial T_{1}} \frac{\partial \tau_{1}}{\partial P_{1}}\right)-Y_{2} \frac{\partial \tau_{1}}{\partial P_{1}}\left(T, P_{1}\right)\left(\frac{\partial \varepsilon_{2}}{\partial P_{2}} \frac{\partial \tau_{2}}{\partial T_{2}}-\frac{\partial \varepsilon_{2}}{\partial T_{2}} \frac{\partial \tau_{2}}{\partial P_{2}}\right)
$$

and that

$$
\operatorname{det}\left(M_{\kappa=1}\right)=\frac{\partial \varepsilon}{\partial P} \frac{\partial \tau}{\partial T}-\frac{\partial \varepsilon}{\partial T} \frac{\partial \tau}{\partial P} .
$$

For a gas mixture, the Lemma B.1 in Appendix B allows to conclude. For a fluid mixture, the Proposition 2.1 allows to apply the point $i i$ of the Lemma B.1 to the equations of state $\tau(T, P)$ and $\varepsilon(T, P)$ of the mixture.

\section{A.1.3. Energy equation}

We easily obtain that

$$
\begin{aligned}
\partial_{t}(\rho E)+\partial_{x}\left[\left(\rho E+P^{\mathrm{eq}}\right) u^{\mathrm{eq}}\right] & =\partial_{x}\left(\lambda \mu^{\mathrm{eq}} u^{\mathrm{eq}} \partial_{x} u^{\mathrm{eq}}\right)-\partial_{x}\left[\lambda u^{\mathrm{eq}}\left(\alpha_{11} P_{1}^{\mathrm{eq}}+\alpha_{21} P_{2}^{\mathrm{eq}}\right)\right] \\
-\partial_{x}\left[\lambda \left(\alpha_{1}^{\mathrm{eq}} P_{1}^{\mathrm{eq}} u_{11}\right.\right. & \left.\left.+\alpha_{2}^{\mathrm{eq}} P_{2}^{\mathrm{eq}} u_{21}\right)\right]-\partial_{x}\left[\lambda \rho\left(Y_{1} \varepsilon_{1}^{\mathrm{eq}} u_{11}+Y_{2} \varepsilon_{2}^{\mathrm{eq}} u_{21}\right)\right] \\
& -\partial_{x}\left[\lambda \rho\left(Y_{1} \varepsilon_{11}+Y_{2} \varepsilon_{21}\right) u^{\mathrm{eq}}\right]-\partial_{x}\left[\lambda \rho\left(Y_{1} u_{11}+Y_{2} u_{21}\right) \frac{\left(u^{\mathrm{eq}}\right)^{2}}{2}\right]+\mathcal{O}\left(\lambda^{2}\right) .
\end{aligned}
$$

We conclude the proof by using the Property A.1 and by noting that $J_{k}=-\rho Y_{k} u_{k 1}$ (cf. Lem. A.1) and that $h_{k} \equiv \varepsilon_{k}+P_{k} / \rho_{k}$.

\section{A.2. Proof of proposition 4.1}

It remains to prove that under the Hypothesis 2.1, the flux $J_{k}=-\rho Y_{k} u_{k 1}$ can be written with (54) and (55) for a gas mixture and with (56) and (57) for a fluid mixture.

We prove the result for a gas mixture. We have

$$
\left\{\begin{array}{l}
Y_{1} \tau_{1}\left(T^{\mathrm{eq}}, P_{1}^{\mathrm{eq}}\right)-\left(1-Y_{1}\right) \tau_{2}\left(T^{\mathrm{eq}}, P_{2}^{\mathrm{eq}}\right)=0 \\
P_{1}^{\mathrm{eq}}+P_{2}^{\mathrm{eq}}=P^{\mathrm{eq}}
\end{array}\right.
$$


Then, $P_{k}^{\mathrm{eq}}$ is a function of $Y_{1}, P^{\mathrm{eq}}$ and $T^{\mathrm{eq}}$ (this is also a consequence of the point $i$ of Th. 2.1). And since $J_{1}=\rho Y_{1} Y_{2}\left(Y_{2} \partial_{x} P_{1}^{\mathrm{eq}}-Y_{1} \partial_{x} P_{2}^{\mathrm{eq}}\right)$, we can write that

$$
J_{1}=\chi_{1} \partial_{x} Y_{1}+\beta_{1} \partial_{x} P^{\mathrm{eq}}+\gamma_{1} \partial_{x} T^{\mathrm{eq}}
$$

where

$$
\left\{\begin{array}{l}
\chi_{1}=\rho Y_{1} Y_{2}\left(Y_{2} \partial_{Y_{1}} P_{1}^{\mathrm{eq}}-Y_{1} \partial_{Y_{1}} P_{2}^{\mathrm{eq}}\right), \\
\beta_{1}=\rho Y_{1} Y_{2}\left(Y_{2} \partial_{P^{\mathrm{eq}}} P_{1}^{\mathrm{eq}}-Y_{1} \partial_{P^{\mathrm{eq}}} P_{2}^{\mathrm{eq}}\right), \\
\gamma_{1}=\rho Y_{1} Y_{2}\left(Y_{2} \partial_{T^{\mathrm{eq}}} P_{1}^{\mathrm{eq}}-Y_{1} \partial_{T^{\mathrm{eq}}} P_{2}^{\mathrm{eq}}\right)
\end{array}\right.
$$

with now $P_{k}^{\mathrm{eq}}=P_{k}^{\mathrm{eq}}\left(Y_{1}, P^{\mathrm{eq}}, T^{\mathrm{eq}}\right)$. Moreover, the system (74) shows that

$$
\left\{\begin{array}{l}
\tau_{1}+\tau_{2}+Y_{1} \frac{\partial \tau_{1}}{\partial P_{1}^{\mathrm{eq}}} \cdot \frac{\partial P_{1}^{\mathrm{eq}}}{\partial Y_{1}}-Y_{2} \frac{\partial \tau_{2}}{\partial P_{2}^{\mathrm{eq}}} \cdot \frac{\partial P_{2}^{\mathrm{eq}}}{\partial Y_{1}}=0 \\
Y_{1} \frac{\tau_{1}}{\partial P_{1}^{\mathrm{eq}}} \cdot \frac{\partial P_{1}^{\mathrm{eq}}}{\partial P^{\mathrm{eq}}}-Y_{2} \frac{\partial \tau_{2}}{\partial P_{2}^{\mathrm{eq}}} \cdot \frac{\partial P_{2}^{\mathrm{eq}}}{\partial P^{\mathrm{eq}}}=0 \\
Y_{1}\left(\frac{\partial \tau_{1}}{\partial P_{1}^{\mathrm{eq}}} \cdot \frac{\partial P_{1}^{\mathrm{eq}}}{\partial T^{\mathrm{eq}}}+\frac{\partial \tau_{1}}{\partial T^{\mathrm{eq}}}\right)-Y_{2}\left(\frac{\partial \tau_{2}}{\partial P_{2}^{\mathrm{eq}}} \cdot \frac{\partial P_{2}^{\mathrm{eq}}}{\partial T^{\mathrm{eq}}}+\frac{\partial \tau_{2}}{\partial T^{\mathrm{eq}}}\right)=0 \\
\frac{\partial P_{1}^{\mathrm{eq}}}{\partial Y_{1}}+\frac{\partial P_{2}^{\mathrm{eq}}}{\partial Y_{1}}=0 \\
\frac{\partial P_{1}^{\mathrm{eq}}}{\partial P^{\mathrm{eq}}}+\frac{\partial P_{2}^{\mathrm{eq}}}{\partial P^{\mathrm{eq}}}=1 \\
\frac{\partial P_{1}^{\mathrm{eq}}}{\partial T^{\mathrm{eq}}}+\frac{\partial P_{2}^{\mathrm{eq}}}{\partial T^{\mathrm{eq}}}=0 .
\end{array}\right.
$$

We can easily invert this linear system which gives the partial derivatives of $P_{k}^{\mathrm{eq}}\left(Y_{1}, P^{\mathrm{eq}}, T^{\mathrm{eq}}\right)$ with respect to $Y_{1}, P^{\mathrm{eq}}$ and $T^{\mathrm{eq}}$. And, some basic calculus give (55).

The proof for a fluid mixture is similar by noting that the equality $Y_{1} \tau_{1}\left(T^{\mathrm{eq}}, P^{\mathrm{eq}}\right)+\left(1-Y_{1}\right) \tau_{2}\left(T^{\mathrm{eq}}, P^{\mathrm{eq}}\right)=\rho^{-1}$ implies that $P^{\mathrm{eq}}=P^{\mathrm{eq}}\left(Y_{1}, \rho, T^{\mathrm{eq}}\right)$.

\section{A.3. A remark}

It is important to underline that, in the case of a fluid mixture, the non conservative terms $P_{\mathrm{int}} \partial_{x} \alpha_{k}$ and $P_{\text {int }} U_{\text {int }} \partial_{x} \alpha_{k}$ in (20) are necessary to obtain the zero order expansions (69) and (73). This implies that these modelling terms - which are supposed to model interfacial phenomena - are necessary to compute the first order Chapman-Enskog expansion and, then, to obtain the transport coefficients.

Let us notice that the closure law (24b) for a fluid mixture is also necessary to obtain the expansion (73).

\section{Appendix B.}

We prove the following lemma:

Lemma B.1. Under the Hypothesis 2.1:

i) The equation of state $\tau_{k}\left(T_{k}, P_{k}\right)$ verifies

$$
\frac{\partial \tau_{k}}{\partial P_{k}}\left(T_{k}, P_{k}\right)<0
$$


ii) The equations of state $\tau_{k}\left(T_{k}, P_{k}\right)$ and $\varepsilon_{k}\left(T_{k}, P_{k}\right)$ verify

$$
\frac{\partial \varepsilon_{k}}{\partial P_{k}} \frac{\partial \tau_{k}}{\partial T_{k}}-\frac{\partial \varepsilon_{k}}{\partial T_{k}} \frac{\partial \tau_{k}}{\partial P_{k}}>0
$$

\section{Proof of Lemma B.1.}

Point i) The second thermodynamic principle (9) shows that

$$
\left\{\begin{array}{l}
\frac{\partial T_{k}}{\partial \tau_{k}}\left(\tau_{k}, s_{k}\right)=-\frac{\partial^{2} \varepsilon_{k}}{\partial \tau_{k} \partial s_{k}}\left(\tau_{k}, s_{k}\right), \\
\frac{\partial T_{k}}{\partial s_{k}}\left(\tau_{k}, s_{k}\right)=-\frac{\partial^{2} \varepsilon_{k}}{\partial s_{k}^{2}}\left(\tau_{k}, s_{k}\right)
\end{array}\right.
$$

and that

$$
\frac{\partial P_{k}}{\partial \tau_{k}}\left(\tau_{k}, T_{k}\right)=-\frac{\partial^{2} \varepsilon_{k}}{\partial \tau_{k}^{2}}\left(\tau_{k}, s_{k}\right)-\frac{\partial^{2} \varepsilon_{k}}{\partial \tau_{k} \partial s_{k}}\left(\tau_{k}, s_{k}\right) \frac{\partial s_{k}}{\partial \tau_{k}}\left(\tau_{k}, T_{k}\right)
$$

by considering the function $P_{k}\left[\tau_{k}, s_{k}\left(\tau_{k}, T_{k}\right)\right]$. Let us now consider the function $T_{k}\left[\tau_{k}, s_{k}\left(\tau_{k}, T_{k}\right)\right]$. We have

$$
\frac{\partial T_{k}}{\partial \tau_{k}}\left(\tau_{k}, s_{k}\right)+\frac{\partial T_{k}}{\partial s_{k}}\left(\tau_{k}, s_{k}\right) \frac{\partial s_{k}}{\partial \tau_{k}}\left(\tau_{k}, T_{k}\right)=0
$$

which implies that

$$
\frac{\partial s_{k}}{\partial \tau_{k}}\left(\tau_{k}, T_{k}\right)=-\frac{\partial^{2} \varepsilon_{k}}{\partial \tau_{k} \partial s_{k}}\left(\tau_{k}, s_{k}\right)\left[\frac{\partial^{2} \varepsilon_{k}}{\partial s_{k}^{2}}\left(\tau_{k}, s_{k}\right)\right]^{-1}
$$

by using (76). Then

$$
\frac{\partial P_{k}}{\partial \tau_{k}}\left(\tau_{k}, T_{k}\right)=-\left[\frac{\partial^{2} \varepsilon_{k}}{\partial \tau_{k}^{2}}\left(\tau_{k}, s_{k}\right) \frac{\partial^{2} \varepsilon_{k}}{\partial s_{k}^{2}}\left(\tau_{k}, s_{k}\right)-\left(\frac{\partial^{2} \varepsilon_{k}}{\partial \tau_{k} \partial s_{k}}\left(\tau_{k}, s_{k}\right)\right)^{2}\right]\left[\frac{\partial^{2} \varepsilon_{k}}{\partial s_{k}^{2}}\left(\tau_{k}, s_{k}\right)\right]^{-1}
$$

because of (77). Moreover, the strict convexity of $s_{k}\left(\tau_{k}, \varepsilon_{k}\right)$ implies that $(c f .[14])$

$$
\frac{\partial^{2} \varepsilon_{k}}{\partial s_{k}^{2}}\left(\tau_{k}, s_{k}\right)>0
$$

and that

$$
\frac{\partial^{2} \varepsilon_{k}}{\partial \tau_{k}^{2}}\left(\tau_{k}, s_{k}\right) \frac{\partial^{2} \varepsilon_{k}}{\partial s_{k}^{2}}\left(\tau_{k}, s_{k}\right)-\left(\frac{\partial^{2} \varepsilon_{k}}{\partial \tau_{k} \partial s_{k}}\left(\tau_{k}, s_{k}\right)\right)^{2}>0 .
$$

Then, these two last inequalities with the equality (78) allow to conclude.

Point ii) Since the Hessian matrice of $s_{k}\left(\tau_{k}, \varepsilon_{k}\right)$ is strictly positive, the differential relation (9) allows to write that the matrice

$$
\left(\begin{array}{cc}
\frac{\partial \varepsilon_{k}}{\partial 1 / T_{k}} & \frac{\partial \varepsilon_{k}}{\partial P_{k} / T_{k}} \\
\frac{\partial \tau_{k}}{\partial 1 / T_{k}} & \frac{\partial \tau_{k}}{\partial P_{k} / T_{k}}
\end{array}\right)
$$

is strictly negative. Then, it is easy to conclude by calculating the determinant. 


\section{Appendix C. Proof of Lemma 4.1}

We prove this lemma for a gas mixture, the proof for a fluid mixture being the same. For a fluid mixture whose each species is an ideal gas, it is easy to obtain that

$$
-T \mathrm{~d} s_{k}=\frac{\mathcal{E}_{k}(T)^{\prime}}{Y_{1} \mathcal{E}_{1}(T)^{\prime}+Y_{2} \mathcal{E}_{2}(T)^{\prime}} \mathrm{d} \varepsilon+\frac{T}{m_{k} \tau} \mathrm{d} \tau
$$

by using (9) and the closure relations (3)-(10). Then, we obtain that

$$
\mathcal{A}=\frac{\rho Y_{1} Y_{2} T}{m_{1} m_{2}} \cdot \frac{m_{2} \mathcal{E}_{2}(T)^{\prime}-m_{1} \mathcal{E}_{1}(T)^{\prime}}{Y_{1} \mathcal{E}_{1}(T)^{\prime}+Y_{2} \mathcal{E}_{2}(T)^{\prime}}
$$

and it is easy to conclude by solving (70) with $\kappa=0$. Then, we can evaluate $P_{11}+P_{21}$ which gives the viscosity $\mu$.

\section{REFERENCES}

[1] Arun In, Numerical evaluation of an energy relaxation method for inviscid real fluids. SIAM J. Sci. Comput. 21 (1999) $340-365$.

[2] R. Abgrall and R. Saurel, A multiphase Godunov method for compressible multifluid and multiphase flows. J. Comput. Phys. 150 (1999) 425-467.

[3] G. Allaire, S. Clerc and S. Kokh, A five-equation model for the simulation of interfaces between compressible fluids. J. Comput. Phys. 181 (2002) 577-616.

[4] P. Andries, Étude d'équations modèles pour la simulation découlements raréfiés. Ph.D. thesis, Pierre and Marie Curie University, Paris VI, France (2000).

[5] R.B. Bird, W.E. Stewart and E.N. Lightfoot, Transport Phenomena, §18.4. John Wiley edition (1960).

[6] G. Chanteperdrix, P. Villedieu and J.P. Vila, Un modèle bifluide compressible pour la simulation numérique d'écoulements diphasiques à phases séparées. Preprint, ONERA report (2002).

[7] F. Coquel and B. Perthame, Relaxation of energy and approximate Riemann solvers for general pressure laws in fluid dynamics. SIAM J. Numer. Anal. 35 (1998) 2223-2249.

[8] S. Chapman and T.G. Cowling, The Mathematical theory of non uniform gases: an account of the kinetic theory of viscosity, thermal conduction and diffusion in gases. Cambridge University Press (1970, reedited in 1990).

[9] J.P. Croisille, Contribution à l'étude théorique et à l'approximation par éléments finis du système hyperbolique de la dynamique des gaz multidimensionnelle et multiespèces. Ph.D. thesis, Pierre and Marie Curie University, Paris VI, France (1990). Cf. also the technical note ONERA No. 1991-3.

[10] S. Dellacherie, Sur le caractère entropique des schémas de relaxation appliqués à une équation d'état non classique. $C$. $R$. Acad. Sci. Paris Sér. I 332 (2001) 765-770.

[11] S. Dellacherie, On the Wang Chang-Uhlenbeck equations. Discrete Contin. Dynam. Systems-Series B 3 (2003) $229-253$.

[12] S. Dellacherie and N. Rency, Relations de fermeture pour le système des équations d'Euler multi-espèces. Construction et étude des schémas de relaxation en multi-espèces et en multi-constituants. CEA report R-5999 (2001).

[13] B. Després, Inégalité entropique pour un solveur conservatif du système de la dynamique des gaz en coordonnées de Lagrange. C. R. Acad. Sci. Paris Sér. I 324 (1997) 1301-1306.

[14] E. Godlewski and P.A. Raviart, Numerical Approximation of Hyperbolic Systems of Conservation Laws. Springer-Verlag, Appl. Math. Sci. 118 (1996).

[15] K.E. Grew and T.L. Ibbs, Thermal diffusion in gases. Cambridge University Press (1952).

[16] F. Lagoutière, Modélisation mathématique et résolution numérique de problèmes de fluides compressibles à plusieurs constituants. Ph.D. thesis, Pierre and Marie Curie University, Paris VI, France (2000).

[17] P. Montarnal and C.W. Shu, Real gas computation using an energy relaxation method and high-order WENO schemes. $J$. Comput. Phys. 148 (1999) 59-80.

[18] W. Mulder, S. Osher and J.A. Sethian, Computing interface motion in compressible gas dynamics. J. Comput. Phys. 100 (1992) 209-228. 
[19] B. Perthame, Boltzmann type schemes for gas dynamics and the entropy property. SIAM J. Numer. Anal. 27 (1990) $1405-1421$.

[20] P. Villedieu and P.A. Mazet, Schémas cinétiques pour les équations d'Euler hors équilibre thermochimique. Édition GauthierVillars, La Recherche Aérospatiale 2 (1995) 85-102.

[21] F. De Vuyst, Schémas non conservatifs et schémas cinétiques pour la simulation numérique d'écoulements hypersoniques non visqueux en déséquilibre thermochimique. Ph.D. thesis, Pierre and Marie Curie University, Paris VI, France (1994).

[22] C.S. Wang Chang and G.E. Uhlenbeck, Transport phenomena in polyatomic gases. Report no. CM-681, University of Michigan Research Report, July (1951).

To access this journal online: www.edpsciences.org 